\title{
Distinguishing charged Higgs bosons from different representations at the $\mathrm{LHC}$
}

\author{
Priyotosh Bandyopadhyay ${ }^{a}$ and Antonio Costantini ${ }^{b}$ \\ ${ }^{a}$ Indian Institute of Technology Hyderabad, \\ Kandi, Sangareddy-502287, Telengana, India \\ ${ }^{b}$ Dipartimento di Matematica e Fisica "Ennio De Giorgi", Università del Salento and INFN-Lecce, \\ Via Arnesano, 73100 Lecce, Italy \\ E-mail: bpriyo@ith.ac.in, antonio.costantini@le.infn.it
}

\begin{abstract}
Extending the Standard Model (SM) scalar sector via the addition of one or more Higgs fields in higher dimensional representations adds one or more charged Higgs bosons to the spectrum. Some of these gauge representations with appropriate hypercharge contain doubly-charged Higgs bosons and can be easily distinguished from the models that contain only singly-charged Higgs bosons. In this study we focus on the more challenging question of distinguishing singly-charged Higgs bosons from different representations, viz. doublets and triplets of the $\mathrm{SU}(2)_{L}$ gauge group. We consider a supersymmetric extension of the $\mathrm{SM}$ with a gauge singlet and an $\mathrm{SU}(2)_{L}$ triplet with $\mathrm{Y}=0$ as a benchmark scenario which has rich phenomenological possibilities due to the presence of a light pseudoscalar associated with the $Z_{3}$ symmetric superpotential. A detailed collider simulation considering all SM backgrounds has been carried out in order to determine which final states are more favourable for observing charged Higgs boson from particular representations as compared with others. We show that different representations can be probed and distinguished via singly-charged Higgs boson phenomenology at the $14 \mathrm{TeV}$ LHC with an earliest data of $\sim 50 \mathrm{fb}^{-1}$ of integrated luminosity.
\end{abstract}

KEYWORDS: Supersymmetry Phenomenology

ARXIV EPRINT: 1710.03110 


\section{Contents}

1 Introduction 1

2 Charged Higgs bosons in various supersymmetric extensions of the SM 3

3 The model 5

4 Triplet-like singly charged Higgs bosons $\quad 6$

$\begin{array}{llr}5 & \text { Benchmark points for the collider study } & 8\end{array}$

6 Final state topologies and simulation at the LHC 11

$\begin{array}{lll}6.12 b+2 \tau & 14\end{array}$

$\begin{array}{lll}6.23 \ell & 15\end{array}$

$\begin{array}{lll}6.3 & 3 \tau & 17\end{array}$

$\begin{array}{lll}6.4 & 4 \ell & 17\end{array}$

$\begin{array}{llr}7 & \text { Reconstruction of the charged Higgs boson } & 18\end{array}$

8 Distinguishing from other extended scenario 21

$\begin{array}{llr}9 & \text { Conclusions } & 23\end{array}$

\section{Introduction}

In 2012 the ATLAS [1] and CMS [2] collaborations announced the discovery of a new elementary particle which was the candidate Higgs boson. This particle was the missing part of the Standard Model (SM) of particle physics. The ATLAS and CMS collaborations found a $125 \mathrm{GeV}$ mass resonance with the properties that are mostly of the SM Higgs boson. The role of the Higgs boson in the SM is to give mass to all the elementary particles, apart from the photon and the gluon, through the mechanism of spontaneous symmetry breaking (SSB). The discovery made at the Large Hadron Collider (LHC) has revealed that the SSB mechanism is realised in a gauge theory such as the SM by at least one Higgs doublet. However, the possible existence of other scalar bosons cannot be excluded. This is mainly due to the not yet measured self interactions of the Higgs boson. Clearly, they are essential in order to establish the mechanism of electroweak symmetry breaking (EWSB), which is crucial in the SM dynamics, with better precision.

In spite of its success in the explanation of the properties of the known elementary particles, the SM is not a completely satisfactory theory. There are, in fact, long-standing issues, such that the gauge-hierarchy problem, the cold dark matter candidate, the masses of the neutrinos etc., to which the SM does not provide a satisfactory answer. 
In this article we are interested in extensions of Higgs sector via various possible gauge representations. In principle these extensions are possible both with and without supersymmetry but in this analysis we consider only the supersymmetric ones. However the Higgs phenomenology and the non-standard decays are similar in both the cases. The interesting fact is that any extension of SM other than with a singlet superfield which takes part in EWSB gives rise to, at least, one physical massive charged Higgs boson. In the case of minimal supersymmetric extension of SM (MSSM) we have two Higgs doublets with opposite hypercharge which results in a physical charged Higgs bosons $h^{ \pm}$. We refer to such a charged Higgs boson as a doublet-type charged Higgs boson and its coupling to SM fermions are given by Yukawa couplings and $\tan \beta$, the ratio of the vevs of the two Higgs doublets [3].

The other possibilities come from the triplet representation of $\mathrm{SU}(2)$. It is possible to include triplet(s) with $Y=0, Y= \pm 1$ or both [4]. Each of them has its own signature, apart from addition of one or more charged Higgs bosons to the spectrum. The simplest extension is with a $Y=0$ triplet which gives rise to two more physical charged Higgs bosons after the EWSB [5,6]. Such extension is constrained by the $\rho$ parameter [7] because the $\mathrm{SU}(2)$ triplet with $Y=0$ hypercharge contributes to the tree-level mass of the $W^{ \pm}$gauge boson but not to the $Z$ one. The vev of the $Y=0$ triplet, which breaks the custodial symmetry, is then restricted to $\lesssim 5 \mathrm{GeV}[5,6]$. Breaking of custodial symmetry leads to the non-standard signature of $h^{ \pm} \rightarrow Z W^{ \pm}$which is very typical of triplet extension $[5,6]$.

As we already mentioned it is also possible to consider triplet(s) with non-zero hypercharge. Such extensions not only lead to triplet-like charged Higgs bosons but also predict the existence of doubly charged Higgs bosons [8]. These extensions also break the custodial symmetry and hence the triplet vevs are also restricted by the $\rho$ parameter, similarly to the $Y=0$ scenario. However a combination of $Y=0$ and $Y= \pm 1$ can restore the custodial symmetry, as was shown by Georgi-Machacek [9] and has also been studied in the supersymmetric context [10].

The charged Higgs boson phenomenology can be largely affected by the existence of a very light scalar ( $C P$-even or $C P$-odd), e.g. the next-to-Minimal Supersymmetric Standard Model (NMSSM) [11]. In this case $h^{ \pm} \rightarrow a_{1} W^{ \pm}$is kinematically allowed for a light charged Higgs boson with mass $\lesssim 200 \mathrm{GeV}$. In a $Z_{3}$ symmetric superpotential, e.g. the NMSSM, such light pseudoscalar is a pseudo Nambu-Goldstone (pNG) boson of a global U(1) symmetry. Such $Z_{3}$ symmetric superpotential in the triplet extensions $[6,8]$ can also lead to the existence of similar light pseudoscalar. Existence of light boson(s) makes the triplet charged Higgs boson phenomenology further more interesting. In this article our main focus is to distinguish triplet-like extensions from the usual doublet-like extensions. We will consider an extension of MSSM with a SM gauge singlet and a $Y=0$ triplet superfields, called the TNMSSM [12]. It gives the possibility to probe both the nonstandard modes of the light charged Higgs boson decays, i.e. $h^{ \pm} \rightarrow a_{1} W^{ \pm}$and $h^{ \pm} \rightarrow Z W^{ \pm}$. The first one is possible due to existence of pNG boson and the second one is due to triplet structure of the light charged Higgs boson.

The triplet-type charged Higgs bosons are different from the doublet-type in two aspects. Firstly they do not couple directly to fermions with the usual Yukawa interaction and secondly they can decay into $Z W^{ \pm}$. So far the searches of the light charged Higgs 
boson at colliders, specially at the LHC, were focused on the doublet-like light charged Higgs [13-16]. The doublet-like charged Higgs boson is obtained through fermionic production modes, viz. $p p \rightarrow t b h^{ \pm} /, g b \rightarrow t, h^{ \pm}$, and then it is searched via $h^{ \pm} \rightarrow \tau \nu / t b$. However, a triplet-like charged Higgs boson will be surely missed in this case, as it does not couple to fermions directly, which suppresses both the production and its fermionic decay modes. In a PYTHIA based analysis we try to distinguish such light doublet- and triplet-type charged Higgs bosons at the LHC.

We organize the paper as follows. In section 2 we give a brief summer of possible extensions of the Higgs sectors where the charged Higgs can come from different representations. Section 3 gives a summer of the triplet/singlet extension of the MSSM. Possible non-standard decays of the triplet-type charged Higgs bosons are discussed in section 4 . In section 5 we treat the phenomenology of triplet- and doublet-like light charged Higgs boson and choose some benchmark points for a collider study at the LHC. In section 6 we perform a detail collider simulation for the signal and consider all the dominant SM backgrounds for the chosen final states, presenting the relative results. In section 7 we present the results for the reconstruction of the charged Higgs mass and in section 8 the correlation among various model with singly and doubly charged Higgs bosons before our conclusions, which are contained in section 9 .

\section{Charged Higgs bosons in various supersymmetric extensions of the SM}

In the Standard Model we do not have any physical charged Higgs boson and this physical state can be achieved by extending the scalar sectors with at least one more $\mathrm{SU}(2)_{L}$ doublet or triplet. We summarize in this section the status of the charged Higgs sector in various models beyond the SM, comparing the number of the charged Higgs bosons and their most important features in terms of allowed decay modes. In table 1 we present the total Higgs spectrum along with the most important feature of the lightest charged Higgs boson in various supersymmetric extensions of the SM.

The MSSM is the most simple supersymmetric theory which allows a single charged Higgs boson in the spectrum. However the charged Higgs boson decays mostly in fermionic modes, viz. $\tau, \nu$ and/or $t, b$. There is an inherent mass degeneracy (nearly) with the pseudoscalar boson $(A)$ and heavy Higgs boson $(H)$ present in the spectrum which prohibits the decays of $h^{ \pm} \rightarrow A / H W^{ \pm}$, as shown in scenario (a). In the simplest extension of MSSM, i.e. the NMSSM, the situation is similar, scenario (b). This is due to the fact that the singlet superfield, after acquiring vev, give rise to an additional scalar and pseudo-scalar in the spectrum but not to a charged Higgs boson. Instead of one, now there are two pseudoscalar bosons. Among which the doublet one will be degenerate with the charged Higgs boson and singlet one can be arbitrary. A very light pseudoscalar can be achieved by considering $Z_{3}$ symmetric superpotential (scenario (c)), which is realized in the limit $A_{i} \rightarrow 0$. In this limit there is an extra U(1) global symmetry, known as the R-symmetry in the literature [11], which is common to supersymmetric theories with only cubic terms in the superpotential (scale invariant). The lightest pseudoscalar of the spectrum is the Nambu-Goldstone mode of this extra symmetry, if $A_{i} \equiv 0$. If the symmetry is softly broken 


\begin{tabular}{|c|c|c|}
\hline $\operatorname{MSSM}(\mathbf{a})$ & NMSSM (b) & $\mathrm{NMSSM}+A_{i} \sim 0(\mathbf{c})$ \\
\hline$H^{ \pm}, A$ degenerate & $H^{ \pm}, a_{1}, a_{2}$ & \multirow{2}{*}{$\begin{array}{c}H^{ \pm} \rightarrow a_{1} W^{ \pm} \text {allowed } \\
1 H^{ \pm}\end{array}$} \\
\hline \multicolumn{2}{|l|}{$1 H^{ \pm}$} & \\
\hline \multicolumn{3}{|c|}{ Triplet Superfield $\hat{T}$} \\
\hline$Y=0(\mathbf{d})$ & $Y= \pm 1(\mathbf{e})$ & $Y=0, \pm 1(\mathbf{f})$ \\
\hline$h_{1}^{ \pm} \not \supset a_{1} W^{ \pm}\left(m_{a_{1}} \gtrsim m_{h_{1}^{ \pm}}\right)$ & $h_{1}^{ \pm} \rightarrow Z W^{ \pm}$allowed & custodial symmetry \\
\hline $3 h_{i}^{ \pm}$ & $3 h_{i}^{ \pm}, 2 h_{j}^{ \pm \pm}$ & $5 h_{i}^{ \pm}, 2 h_{j}^{ \pm \pm}$ \\
\hline \multicolumn{3}{|c|}{$\mathrm{MSSM}+\hat{S}+\hat{T}$ with $\mathrm{Y}=0+A_{i} \sim 0(\mathrm{~g})$} \\
\hline \multicolumn{3}{|c|}{$h_{1}^{ \pm}$triplet-type } \\
\hline \multirow{2}{*}{\multicolumn{3}{|c|}{$\begin{aligned} h_{1}^{ \pm} \rightarrow & Z W^{ \pm} \text {enhanced for } \lambda_{T} \sim 0 \\
& h_{1}^{ \pm} \rightarrow a_{1} W^{ \pm} \text {allowed }\end{aligned}$}} \\
\hline & & \\
\hline \multicolumn{3}{|c|}{$3 h_{i}^{ \pm}$} \\
\hline
\end{tabular}

Table 1. The charged Higgs sector in various SUSY theories.

the pseudoscalar takes the role of an axion-like particle. Having a very low mass the decay $H^{ \pm} \rightarrow a_{1} W^{ \pm}$is kinematically allowed, however such light pseudoscalar faces direct and indirect constraints from LEP [17, 18] and other experiments $[19,20]$.

Scenarios (d), (e) and (f) correspond to $Y=0, Y= \pm 1$ and $Y=0, \pm 1$ (GeorgiMachacek) cases where one or more $\mathrm{SU}(2)_{L}$ triplet superfields are added to the MSSM superfield content. In the simplified extension of (d), where a $Y=0$ triplet superfield is added to the MSSM, there are two more singly charged Higgs boson in the spectrum respect to the MSSM. The triplet charged Higgs bosons do not couple directly to the fermions, which makes their production at the LHC rather difficult, their decay to fermions is also suppressed and non-standard decays come into the picture [21, 22]. However, in this scenario the two-body decay $h_{1}^{ \pm} \rightarrow a_{1} W^{ \pm}$is not allowed because $m_{h_{1}^{ \pm}} \lesssim m_{a_{1}}[21,22]$. The most important decay mode is then $h_{1}^{ \pm} \rightarrow Z W^{ \pm}$. This interaction is present at tree-level in theories with scalar triplets which acquire vevs and break custodial symmetry at the tree-level. Instead, if the model has scalar doublets or singlets of $\mathrm{SU}(2)_{L}$ the breaking of the custodial symmetry is only possible at loop level and so is the decay $h_{i}^{ \pm} \rightarrow Z W^{ \pm}$. If the triplet superfield has $Y= \pm 1$ hypercharge, (scenario (e)), then in the spectrum, we will see a doubly charged Higgs bosons along with two additional triplet-type singly charged Higgs bosons [8]. Finally scenario (f) has both a $Y=0$ and a $Y= \pm 1$ triplet superfields and the corresponding charged Higgs bosons. This is the supersymmetric version of the well-known Georgi-Machacek model [9]. The most important feature of scenario (f) is that the custodial symmetry can be naturally imposed [10].

Scenario $(\mathrm{g})$ is considered in [23], where on the top of the MSSM superfield content, there is a singlet superfield and a triplet superfield with $Y=0$. The superpotential is $Z_{3}$ symmetric, meaning that only cubic terms are allowed. In the limit $A_{i} \sim 0$, it exhibits a softly broken U(1) symmetry, similarly to scenario (c) and the lightest pseudoscalar is 
the pseudo Nambu-Goldstone mode of this extra $\mathrm{U}(1)$ symmetry that can be very light. In this scenario the lightest charged Higgs boson can decay in $a_{1} W^{ \pm}$and $Z W^{ \pm}$and if it is triplet-like its decay into fermions is suppressed. Below we briefly introduce the model which will be used later for the phenomenological studies to distinguish among doublet and triplet charged Higgs boson(s) at the LHC.

\section{The model}

We consider an extension of the MSSM with a (gauge) singlet superfield $\hat{S}$ and a triplet superfield $\hat{T}$ with $Y=0$. The model is detailed in [12] and here we will give the very basic properties. The Higgs superfields are given below,

$$
\hat{T}=\left(\begin{array}{cc}
\sqrt{\frac{1}{2}} \hat{T}^{0} & \hat{T}_{2}^{+} \\
\hat{T}_{1}^{-} & -\sqrt{\frac{1}{2}} \hat{T}^{0}
\end{array}\right), \quad \hat{H}_{u}=\left(\begin{array}{c}
\hat{H}_{u}^{+} \\
\hat{H}_{u}^{0}
\end{array}\right), \quad \hat{H}_{d}=\left(\begin{array}{c}
\hat{H}_{d}^{0} \\
\hat{H}_{d}^{-}
\end{array}\right), \quad \hat{S},
$$

where $\hat{T}^{0}$ is a complex neutral superfield, while $\hat{T}_{1}^{-}$and $\hat{T}_{2}^{+}$are the charged Higgs superfields. $\hat{H}_{u}$ and $\hat{H}_{d}$ are the usual doublet superfields of the MSSM and $\hat{S}$ is the singlet superfield. The gauge symmetry implies that the Yukawa interactions are identical to the MSSM ones, because neither the singlet nor the triplet superfields have any interaction with the fermionic superfields. This means that the superpotential can be written as

$$
\hat{W}_{\mathrm{TNMSSM}}=\hat{W}_{\mathrm{MSSM}}+\hat{W}_{T S}
$$

with

$$
\hat{W}_{\mathrm{MSSM}}=y_{t} \hat{U} \hat{H}_{u} \cdot \hat{Q}-y_{b} \hat{D} \hat{H}_{d} \cdot \hat{Q}-y_{\tau} \hat{E} \hat{H}_{d} \cdot \hat{L},
$$

being the superpotential of the MSSM, while

$$
\hat{W}_{T S}=\lambda_{T} \hat{H}_{d} \cdot \hat{T} \hat{H}_{u}+\lambda_{S} \hat{S} \hat{H}_{d} \cdot \hat{H}_{u}+\frac{\kappa}{3} \hat{S}^{3}+\lambda_{T S} \hat{S} \operatorname{Tr}\left[\hat{T}^{2}\right]
$$

Here "." denotes a contraction with the Levi-Civita symbol $\epsilon^{i j}$, with $\epsilon^{12}=+1$.

It is a characteristic of any scale invariant supersymmetric theory with a cubic superpotential that the complete Lagrangian with the soft SUSY breaking terms has an accidental $Z_{3}$ symmetry. This is generated by the invariance of all of its components after multiplication of the chiral superfields by the phase $e^{2 \pi i / 3}$. This $Z_{3}$ symmetry is promoted to a global U(1) symmetry in the limit $A_{i} \rightarrow 0$, where the $A_{i}$ are the trilinear terms of the softbreaking part of the scalar potential [12]. This global U(1) symmetry can be softly broken by small $A_{i}$, giving rise to a very light pseudoscalar which is the pseudo Nambu-Goldstone mode of the symmetry.

We assume that all the coefficients involved in the Higgs sector are real in order to preserve CP invariance. The breaking of the $\mathrm{SU}(2)_{L} \times \mathrm{U}(1)_{Y}$ electroweak symmetry is then obtained by giving real vevs to the neutral components of the Higgs field

$$
<H_{u}^{0}>=\frac{v_{u}}{\sqrt{2}}, \quad<H_{d}^{0}>=\frac{v_{d}}{\sqrt{2}}, \quad<S>=\frac{v_{S}}{\sqrt{2}}, \quad<T^{0}>=\frac{v_{T}}{\sqrt{2}},
$$


which give mass to the $W^{ \pm}$and $Z$ bosons

$$
m_{W}^{2}=\frac{1}{4} g_{L}^{2}\left(v^{2}+4 v_{T}^{2}\right), \quad m_{Z}^{2}=\frac{1}{4}\left(g_{L}^{2}+g_{Y}^{2}\right) v^{2}, \quad v^{2}=\left(v_{u}^{2}+v_{d}^{2}\right), \quad \tan \beta=\frac{v_{u}}{v_{d}},
$$

and also induce, as mentioned above, a $\mu$-term of the form $\mu_{D}=\frac{\lambda_{S}}{\sqrt{2}} v_{S}+\frac{\lambda_{T}}{2} v_{T}$. The triplet vev $v_{T}$ is strongly constrained by the global fit on the measurement of the $\rho$ parameter [7]

$$
\rho=1.0004_{-0.0004}^{+0.0003}
$$

which restricts its value to $v_{T} \leq 5 \mathrm{GeV}$. Respect to the tree-level expression, the non-zero triplet contribution to the $W^{ \pm}$mass leads to a deviation of the $\rho$ parameter

$$
\rho=1+4 \frac{v_{T}^{2}}{v^{2}}
$$

\section{Triplet-like singly charged Higgs bosons}

The lightest triplet-like charged Higgs in TNMSSM $[12,23,24]$ can decay to $Z W^{ \pm}$as well as to $a_{1} W^{ \pm}$. Establishing these two non-standard decay modes will be sufficient to prove the existence of higher representations $\mathrm{SU}(2)_{L}$ in the Higgs spectrum, i.e. the triplet as well as the SM gauge singlet. As we already point out in section 2 that in the case of a triplet with non-zero hypercharge, there is a doubly-charged Higgs boson in the spectrum. Its phenomenology have been studied extensively in the literature [25-28]. In this article our goal is to distinguish between doublet- and triplet-like charged Higgs boson by searching for singly charged Higgs bosons at the LHC in appropriate decay channels.

The phenomenology of the lightest charged Higgs boson of the TNMSSM is affected by the presence of a light pseudoscalar, which induces a new decay mode $h_{1}^{ \pm} \rightarrow a_{1} W^{ \pm}$. Along with the existence of the light pseudoscalar, the triplet-like charged Higgs boson adds another tree-level decay mode $h_{1}^{ \pm} \rightarrow Z W^{ \pm}$, which is not possible otherwise. In particular, a $Y=0$ triplet-like charged Higgs boson can decay into $Z W^{ \pm}$, which is a signature of custodial symmetry breaking. If the model has only doublets of $\mathrm{SU}(2)_{L}$ then the decay $h^{ \pm} \rightarrow Z W^{ \pm}$is loop-induced only. Apart from that, the usual doublet-like decay modes into $\tau \nu$ and $t b$ are present via the mixing with the doublets. We summarize the different possible decay modes in the following paragraphs.

The trilinear couplings with charged Higgs bosons, scalar (pseudoscalar) Higgs bosons and $W^{ \pm}$are given by

$$
\begin{aligned}
& g_{h_{i}^{ \pm} W \mp h_{j}}=\frac{i}{2} g_{L}\left(\mathcal{R}_{j 2}^{S} \mathcal{R}_{i 3}^{C}-\mathcal{R}_{j 1}^{S} \mathcal{R}_{i 1}^{C}+\sqrt{2} \mathcal{R}_{j 4}^{S}\left(\mathcal{R}_{i 2}^{C}+\mathcal{R}_{i 4}^{C}\right)\right), \\
& g_{h_{i}^{ \pm} W^{\mp} a_{j}}=\frac{g_{L}}{2}\left(\mathcal{R}_{j 1}^{P} \mathcal{R}_{i 1}^{C}+\mathcal{R}_{j 2}^{P} \mathcal{R}_{i 3}^{C}+\sqrt{2} \mathcal{R}_{j 4}^{P}\left(\mathcal{R}_{i 2}^{C}-\mathcal{R}_{i 4}^{C}\right)\right),
\end{aligned}
$$

where $\mathcal{R}^{S}, \mathcal{R}^{P}, \mathcal{R}^{C}$ are the mixing matrix corresponding to scalar, pseudoscalar and charged Higgs bosons respectively, with $\mathcal{R}_{j 4}^{S}, \mathcal{R}_{j 4}^{P}, \mathcal{R}_{j, 2}^{C}, \mathcal{R}_{j 4}^{C}$ being the triplet part of the mixing matrices [23].

Both the triplet and doublet have $\mathrm{SU}(2)_{L}$ charge and hence they couple to $W^{ \pm}$boson. However the $W^{ \pm}$boson is in the triplet representation of $\mathrm{SU}(2)_{L}$ and this means that in 
the $W^{ \pm} h_{i} h_{j}^{\mp}$ coupling the neutral and charged Higgs bosons have to be doublet(triplet) and triplet-(doublet-)type respectively in order to maintain the gauge invariance.

The decay width of a massive charged Higgs boson in a $W$ boson and a scalar (or pseudoscalar) boson is given by

$$
\Gamma_{h_{i}^{ \pm} \rightarrow W^{ \pm} h_{j} / a_{j}}=\frac{G_{F}}{8 \sqrt{2} \pi} m_{W^{ \pm}}^{2}\left|g_{h_{i}^{ \pm} W^{\mp} h_{j} / a_{j}}\right|^{2} \sqrt{\lambda\left(1, x_{W}, x_{h_{j} / a_{j}}\right)} \lambda\left(1, y_{h_{i}^{ \pm}}, y_{h_{j} / a_{j}}\right)
$$

where $x_{W, h_{j}}=\frac{m_{W, h_{j}}^{2}}{m_{h_{i}^{ \pm}}^{2}}$ and $y_{h_{i}^{ \pm}, h_{j}}=\frac{m_{h_{i}^{ \pm}, h_{j}}^{2}}{m_{W^{ \pm}}^{2}}$ and similarly for $a_{j}$. In TNMSSM this decay channel is prominent for a light charged Higgs boson and it is the dominant decay mode if the charged Higgs boson is triplet-like, because of the suppression of the fermionic couplings.

In theories with $Y=0, \pm 2$ hypercharge triplets, which generally break the custodial symmetry, there is a tree-level interactions $h_{i}^{ \pm}-W^{\mp}-Z$ [4]. In the TNMSSM this coupling is given by

$$
g_{h_{i}^{ \pm} W^{\mp} Z}=-\frac{i}{2}\left(g_{L} g_{Y}\left(v_{u} \sin \beta \mathcal{R}_{i 1}^{C}-v_{d} \cos \beta \mathcal{R}_{i 3}^{C}\right)+\sqrt{2} g_{L}^{2} v_{T}\left(\mathcal{R}_{i 2}^{C}+\mathcal{R}_{i 4}^{C}\right)\right),
$$

where the explicit definition of the rotation angles can be found in [23]. The on-shell decay width is given by

$$
\Gamma_{h_{i}^{ \pm} \rightarrow W^{ \pm} Z}=\frac{G_{F} \cos ^{2} \theta_{W}}{8 \sqrt{2} \pi} m_{h_{i}^{ \pm}}^{3}\left|g_{h_{i}^{ \pm} W \mp Z}\right|^{2} \sqrt{\lambda\left(1, x_{W}, x_{Z}\right)}\left(8 x_{W} x_{Z}+\left(1-x_{W}-x_{Z}\right)^{2}\right),
$$

where $\lambda(x, y, z)=(x-y-z)^{2}-4 y z$ and $x_{Z, W}=\frac{m_{Z, W}^{2}}{m_{h_{i}^{ \pm}}^{2}}[29]$. As it is extensively explained in [23], this decay channel is enhanced for a triplet-like charged Higgs boson in the limit $\lambda_{T} \sim 0$ due to the same sign values of $\mathcal{R}_{12}^{C}$ and $\mathcal{R}_{14}^{C}$.

Beside the non-zero $h_{i}^{ \pm}-W^{\mp}-Z$ coupling at the tree-level due to custodial symmetry breaking, the charged Higgs bosons can also decay into fermions through the Yukawa interaction given below

$$
g_{h_{i}^{+} \bar{u} d}=i\left(y_{u} \mathcal{R}_{i 1}^{C} \mathrm{P}_{\mathrm{L}}+y_{d} \mathcal{R}_{i 3}^{C} \mathrm{P}_{\mathrm{R}}\right),
$$

governed by doublet part of the charged Higgses. The decay width at leading order is

$$
\begin{aligned}
\Gamma_{h_{i}^{ \pm} \rightarrow u d}=\frac{3}{4} \frac{G_{F}}{\sqrt{2} \pi} m_{h_{i}^{ \pm}} \sqrt{\lambda\left(1, x_{u}, x_{d}\right)} & {\left[\left(1-x_{u}-x_{d}\right)\left(\frac{m_{u}^{2}}{\sin ^{2} \beta}\left(\mathcal{R}_{i 1}^{C}\right)^{2}+\frac{m_{d}^{2}}{\cos ^{2} \beta}\left(\mathcal{R}_{i 3}^{C}\right)^{2}\right)\right.} \\
& \left.-4 \frac{m_{u}^{2} m_{d}^{2}}{m_{h_{i}^{ \pm}}^{2}} \frac{\mathcal{R}_{i 1}^{C} \mathcal{R}_{i 3}^{C}}{\sin \beta \cos \beta}\right]
\end{aligned}
$$

where $x_{u, d}=\frac{m_{u, d}^{2}}{m_{h_{i}^{ \pm}}^{2}}$. The decay of the charged Higgs bosons into quarks is suppressed in the case of triplet-like eigenstates, where $\mathcal{R}_{i 1, i 3}^{C} \ll 1$. 


\section{Benchmark points for the collider study}

In this section we select four benchmark points to perform the collider study and to distinguish doublet- and triplet-like points at the LHC. It is evident from eq. (3.4) that the triplet states do not couple to the fermions, which makes their production at the LHC or earlier colliders very difficult as essentially all of them are fermionic colliders. Triplet does not have any $\mathrm{SU}(3)$ charge, thus normal gluon fusion at the LHC cannot produce it. The same property explains why their decays in fermionic modes or fermion induced modes $(\gamma \gamma, g g)$ are also suppressed. In [24] we considered the most relevant experimental bounds to find out the allowed parameter regions. From table 2 we can see apart from the $\sim 125 \mathrm{GeV}$ Higgs boson the next heavier states are mostly triplets and also for all the benchmark points there is a mostly singlet like pseudoscalar, which affects their branching fraction by generating new modes $h_{i} \rightarrow a_{1} a_{1}$ (for neutral Higgs bosons) and $h_{i}^{ \pm} \rightarrow a_{1} W^{ \pm}$ (for charged Higgs bosons). This implies that probing the heavier states will require either searches in new final states and/or higher luminosities.

In table 2 we show the mass spectrum of the selected benchmark points. Together with the recent Higgs data, we have also considered the recent bounds on the stop and sbottom masses [37, 38] and the mass bounds on the lightest chargino from LEP [39]. The recent bounds on the charged Higgs boson mass from both CMS [40,41] and ATLAS [42, 43] are also taken into account. These bounds have been derived in their searches for the light charged Higgs bosons from the decay of a top quark and/or via charged Higgs production in pairs/association, and its decays to $\tau \bar{\nu}$. Addition of any new decay mode will further lower the lower bound for the charged mass exclusion.

The branching fractions of the SM-like Higgs boson for the four benchmark points are presented in table 3 . They are consistent with the observed branching fraction at $2 \sigma$ level. The decay channels of the light pseudoscalar $a_{1}$ are presented in table 4 . Even though they are mostly singlet like, they decay to fermions via mixing with the doublets. The benchmark points are chosen with different dominant decay modes of the charged Higgs bosons. The mostly triplet-like charged Higgs would dominantly decays into $Z W^{ \pm}$. Similarly for some points it can decay to $a_{1} W^{ \pm}$and for mostly doublet-like charged Higgs boson it decays to $t b$.

In table 5 presents the two-body decay branching fractions of the charged Higgs for the benchmark points. We can see that for BP1, the decay branching fraction to $a_{1} W^{ \pm}$is the highest. BP2 seems to have decent decay rates into $h_{125} W^{ \pm}, Z W^{ \pm}$and into $t b$ making it a mixed point where both doublet and triplet natures manifest. In contrary to BP1, BP3 is a doublet-like points which mostly decays to $t b$. Finally, BP4 is characterized by a single dominant decay channel, $h_{1}^{ \pm} \rightarrow Z W^{ \pm}$with a branching fraction of 0.994 . We will see later in the section how we can distinguish such doublet- and triplet-like points. The color code used in table 2 is green to represent a triplet-like charged Higgs boson and red for the doublet-like. We see that for BP3 the charged Higgs boson is mostly doublet and so it is in red. Similarly blue color has been used to signify singlet-like light pseudoscalar boson.

Figure 1 describes a correlation plot for $\mathcal{B} r\left(h_{1}^{ \pm} \rightarrow a_{1} W^{ \pm}\right)$and $\mathcal{B} r\left(h_{1}^{ \pm} \rightarrow Z W^{ \pm}\right)$for the light charged Higgs boson $\left(h_{1}^{ \pm}\right)$. The red points are doublet-like charged Higgs, the green 


\begin{tabular}{|c|c|c|c|c|}
\hline $\begin{array}{c}\text { Benchmark } \\
\text { Points }\end{array}$ & BP1 & BP2 & BP3 & BP4 \\
\hline$m_{h_{1}}$ & $\sim 125$ & $\sim 125$ & $\sim 125$ & $\sim 125$ \\
\hline$m_{h_{2}}$ & 316.14 & 340.44 & 272.87 & 174.21 \\
\hline$m_{h_{3}}$ & 522.41 & 382.56 & 358.12 & 1027.3 \\
\hline$m_{h_{4}}$ & 673.45 & 514.16 & 2094.4 & 1547.7 \\
\hline$m_{a_{1}}$ & 41.221 & 36.145 & 30.655 & 61.537 \\
\hline$m_{a_{2}}$ & 181.34 & 428.68 & 278.22 & 1052.7 \\
\hline$m_{a_{3}}$ & 559.32 & 519.01 & 2149.4 & 1325.9 \\
\hline$m_{h_{1}^{ \pm}}$ & 179.69 & 339.97 & 289.51 & 174.11 \\
\hline$m_{h_{2}^{ \pm}}$ & 316.20 & 399.84 & 2089.7 & 1032.9 \\
\hline$m_{h_{3}^{ \pm}}$ & 535.21 & 519.02 & 2144.9 & 1325.9 \\
\hline
\end{tabular}

Table 2. Benchmark points for a collider study consistent with the $\sim 125 \mathrm{GeV}$ Higgs mass, where the $h_{i=1,2,3,4}, a_{i=1,2,3}$ are at one-loop and $h_{i=1,2,3}^{ \pm}$masses are calculated at tree level. We color in red the states which are mostly doublets $(>90 \%)$ and in blue and green those which are mostly singlet and triplet $(>90 \%)$ respectively. The points are consistent with the $2 \sigma$ limits of $h_{125} \rightarrow W W^{*}, Z Z^{*}, \gamma \gamma[30-36]$.

\begin{tabular}{|c|c|c|c|c|}
\hline \multirow{2}{*}{$\begin{array}{c}\text { Benchmark } \\
\text { Points }\end{array}$} & \multicolumn{4}{|c|}{ Branching ratios } \\
\cline { 2 - 5 } & $a_{1} a_{1}$ & $W^{ \pm} W^{\mp}$ & $Z Z$ & $b \bar{b}$ \\
\hline BP1 & 0.105 & 0.148 & 0.020 & 0.686 \\
\hline BP2 & 0.045 & 0.143 & 0.019 & 0.748 \\
\hline BP3 & 0.052 & 0.115 & 0.015 & 0.770 \\
\hline BP4 & 0.057 & 0.129 & 0.017 & 0.752 \\
\hline
\end{tabular}

Table 3. Relevant decay branching ratios of $h_{125}$ for the benchmark points.

\begin{tabular}{|c|c|c|c|}
\hline \multirow{2}{*}{$\begin{array}{c}\text { Benchmark } \\
\text { Points }\end{array}$} & \multicolumn{3}{|c|}{ Branching ratios(\%) } \\
\cline { 2 - 4 } & $b \bar{b}$ & $\tau \bar{\tau}$ & $\mu \bar{\mu}$ \\
\hline BP1 & 0.942 & $5.77 \times 10^{-2}$ & $2.06 \times 10^{-4}$ \\
\hline BP2 & 0.942 & $5.80 \times 10^{-2}$ & $2.07 \times 10^{-4}$ \\
\hline BP3 & 0.941 & $5.85 \times 10^{-2}$ & $2.09 \times 10^{-4}$ \\
\hline BP4 & 0.943 & $5.72 \times 10^{-2}$ & $2.03 \times 10^{-4}$ \\
\hline
\end{tabular}

Table 4. Decay branching ratios of $a_{1}$ for the benchmark points $B P_{i}$. The kinematically forbidden decays are marked with dashes. 


\begin{tabular}{|c|c|c|c|c|c|c|}
\hline \multirow{2}{*}{$\begin{array}{c}\text { Benchmark } \\
\text { Points }\end{array}$} & \multicolumn{7}{|c|}{ Branching ratios } \\
\cline { 2 - 7 } & $a_{1} W^{ \pm}$ & $h_{125} W^{ \pm}$ & $Z W^{ \pm}$ & $t b$ & $\tau \nu_{\tau}$ & $\mu \nu_{\mu}$ \\
\hline BP1 & 0.969 & $5.56 \times 10^{-5}$ & $2.09 \times 10^{-2}$ & $9.58 \times 10^{-3}$ & $1.29 \times 10^{-4}$ & $4.57 \times 10^{-7}$ \\
\hline BP2 & $3.01 \times 10^{-2}$ & 0.213 & 0.236 & 0.520 & $8.87 \times 10^{-5}$ & $3.15 \times 10^{-7}$ \\
\hline BP3 & $6.43 \times 10^{-2}$ & $3.51 \times 10^{-3}$ & $1.94 \times 10^{-10}$ & 0.932 & $9.25 \times 10^{-5}$ & $3.28 \times 10^{-7}$ \\
\hline BP4 & $1.03 \times 10^{-5}$ & $3.34 \times 10^{-3}$ & 0.994 & - & $1.60 \times 10^{-3}$ & $5.68 \times 10^{-6}$ \\
\hline
\end{tabular}

Table 5. Decay branching ratios of $h_{1}^{ \pm}$for the benchmark points. The kinematically forbidden decays are marked with a dash.

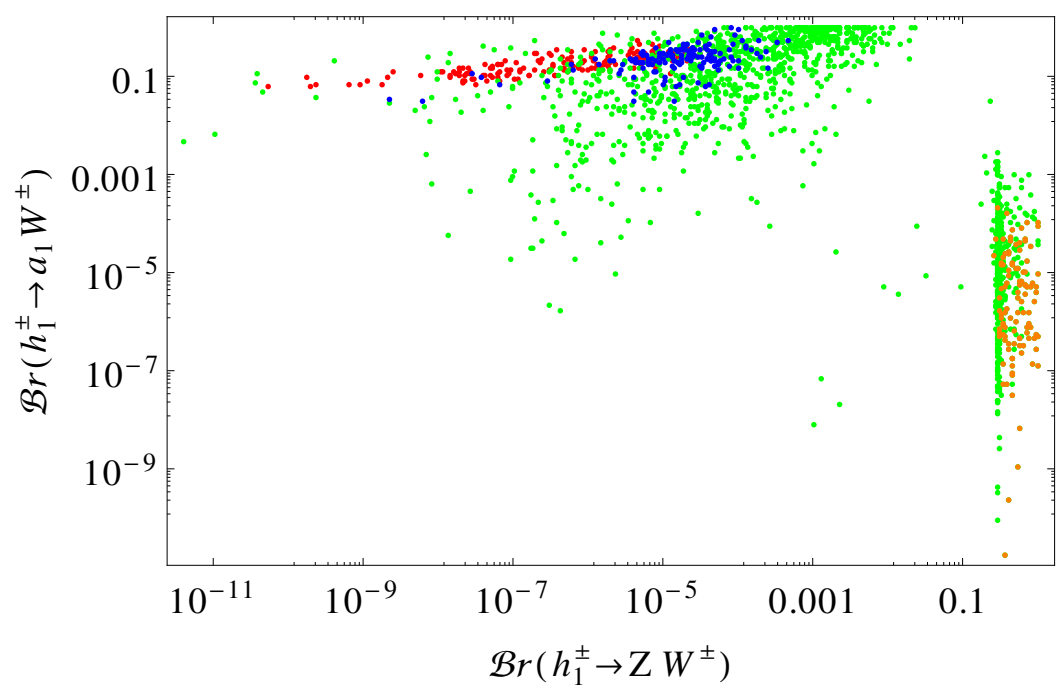

Figure 1. Correlation plot of the branching ratios for the non-standard decays $h_{1}^{ \pm} \rightarrow Z W^{ \pm}$and $h_{1}^{ \pm} \rightarrow a_{1} W^{ \pm}$. The red points are doublet-like charged Higgs, the green ones are triplet-like and the blue ones are mixed-one. The orange ones are characterized by $\lambda_{T} \sim 0$ [23]. We can see that these two decay channels are almost mutually excluded.

ones are triplet-like and the blue ones are mixed-one. The orange ones are characterized by $\lambda_{T} \sim 0$ [23]. We can see that $h_{1}^{ \pm} \rightarrow a_{1} W^{ \pm}$and $h_{1}^{ \pm} \rightarrow Z W^{ \pm}$decay channels are almost mutually excluded and it would be very difficult to probe both the triplet nature of the charged Higgs boson as well as the existence of the light pseudoscalar $a_{1}$ for the same benchmark point.

We have calculated the light charged Higgs production cross-sections in pairs and in association of other particles at the LHC for these benchmark points. For this purpose we have implemented the model in SARAH [44] and we have generated the model files for CalcHEP [45]. The cross-sections have been calculated at the tree-level via Calchep_3.6.23 [45] and table 6 presents the cross-sections which include the associated $\mathrm{K}$-factors $[46,47]$. We can see that for $\mathrm{BP} 1, h_{1}^{ \pm}$is degenerate with $a_{2}$, and for BP2 and $\mathrm{BP} 4, h_{1}^{ \pm}$is degenerate with $h_{2}$ (see table 2). The cross-section contributions from such associated charged Higgs production processes are the dominant ones for the respective benchmark points. 


\begin{tabular}{|c|c|c|c|c|}
\cline { 2 - 5 } \multicolumn{1}{c|}{} & \multicolumn{4}{c|}{ Cross section in fb } \\
\cline { 2 - 5 } \multicolumn{1}{c|}{} & BP1 & BP2 & BP3 & BP4 \\
\hline$h_{1}^{ \pm} h_{1}^{\mp}$ & 148.00 & 13.00 & 12.48 & 166.50 \\
\hline$h_{125} h_{1}^{ \pm}$ & $6.93 \times 10^{-4}$ & $1.82 \times 10^{-2}$ & 0.35 & 0.15 \\
\hline$a_{1} h_{1}^{ \pm}$ & $2.14 \times 10^{-2}$ & $2.48 \times 10^{-3}$ & 5.12 & $9.68 \times 10^{-7}$ \\
\hline$h_{2} h_{1}^{ \pm}$ & 0.28 & 26.42 & 13.89 & 334.62 \\
\hline$a_{2} h_{1}^{ \pm}$ & 292.45 & $2.38 \times 10^{-3}$ & 12.54 & $7.07 \times 10^{-8}$ \\
\hline$Z h_{1}^{ \pm}$ & $1.44 \times 10^{-3}$ & $3.08 \times 10^{-2}$ & $2.66 \times 10^{-8}$ & 0.33 \\
\hline$W^{\mp} h_{1}^{ \pm}$ & $2.08 \times 10^{-2}$ & 0.17 & 166.21 & 0.88 \\
\hline$t h_{1}^{ \pm}$ & $8.13 \times 10^{-2}$ & 3.50 & $4.48 \times 10^{3}$ & 7.60 \\
\hline$t b h_{1}^{ \pm}$ & $3.28 \times 10^{-2}$ & 0.21 & 386.32 & 3.81 \\
\hline
\end{tabular}

Table 6. Production cross-section of the charged Higgs boson $h_{1}^{ \pm}$in various channels at the LHC for a center of mass energy of $14 \mathrm{TeV}$ for the three benchmark points. A K-factor of 1.6 has been used.

\section{Final state topologies and simulation at the LHC}

The TNMSSM can have a light pseudoscalar $a_{1}$ with $m_{a_{1}} \lesssim 60 \mathrm{GeV}$ (see table 2 ). The existence of such light pseudoscalar opens a new mode in the decay of the light charged Higgs boson, i.e. $h_{1}^{ \pm} \rightarrow a_{1} W^{ \pm}$, and the light pseudoscalar boson can further decay into $\tau$ or $b$ pairs. The other possible signature comes from the triplet nature of the light charged Higgs boson, which prompts the $h_{1}^{ \pm} \rightarrow Z W^{ \pm}$decay. For the chosen benchmark points we will focus on these non-standard decays of the charged Higgs boson, which is the result of the existence of a singlet and triplet scalar in the spectrum. For this purpose CalcHEP [45] has been used to generate the decay file SLHA, containing the decay branching ratios and the corresponding mass spectra. The generated events have then been simulated with PYTHIA [48] via the SLHA interface [49, 50]. The simulation at hadronic level has been performed using the Fastjet-3.0.3 [51] with the CAMBRIDGE AACHEN algorithm. We have selected a jet size $R=0.5$ for the jet formation, with the following criteria:

- the calorimeter coverage is $|\eta|<4.5$,

- the minimum transverse momentum of the jet $p_{T, \min }^{\mathrm{jet}}=10 \mathrm{GeV}$ and jets are ordered in $p_{T}$,

- leptons $(\ell=\mathrm{e}, \mu)$ are selected with $p_{T} \geq 10 \mathrm{GeV}$ and $|\eta| \leq 2.5$,

- no jet should be accompanied by a hard lepton in the event,

- $\Delta R_{l j} \geq 0.4$ and $\Delta R_{l l} \geq 0.2$,

- Since an efficient identification of the leptons is crucial for our study, we additionally require a hadronic activity within a cone of $\Delta R=0.3$ between two isolated leptons to be $\leq 0.15 p_{T}^{\ell} \mathrm{GeV}$, with $p_{T}^{\ell}$ the transverse momentum of the lepton, in the specified cone. 


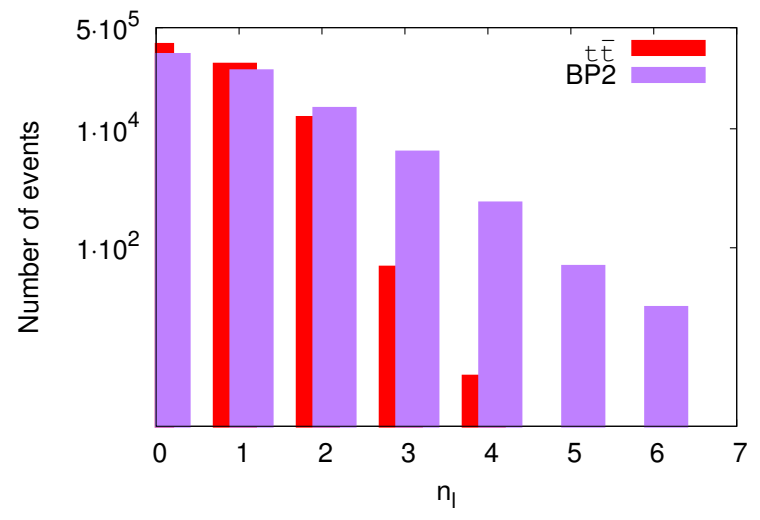

(a)

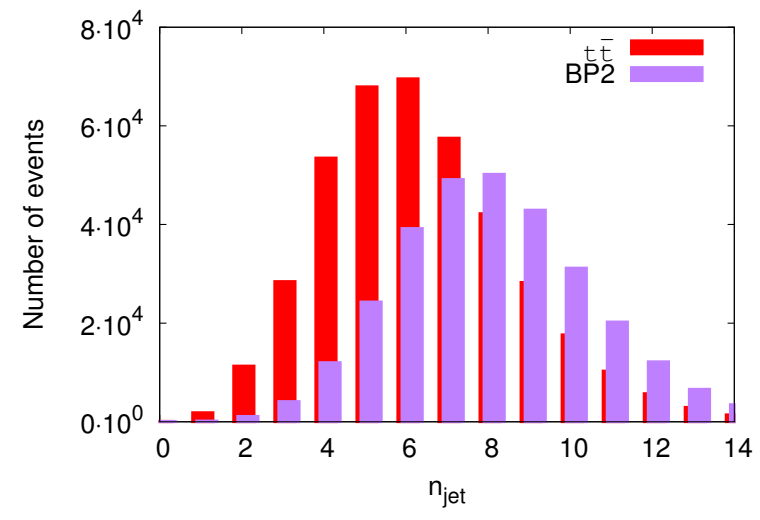

(b)

Figure 2. Jet and lepton multiplicity distributions from BP2 of the $h_{1}^{ \pm} h_{1}^{\mp}$ signal and the SM background $t \bar{t}$ are shown in (a) and (b) respectively.

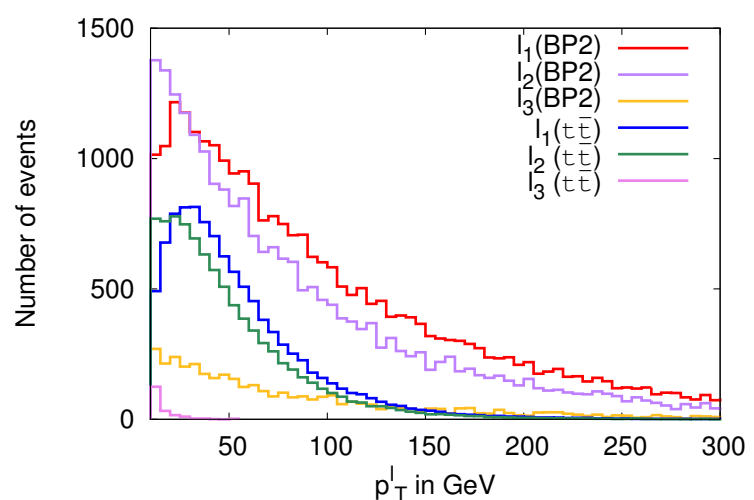

(a)

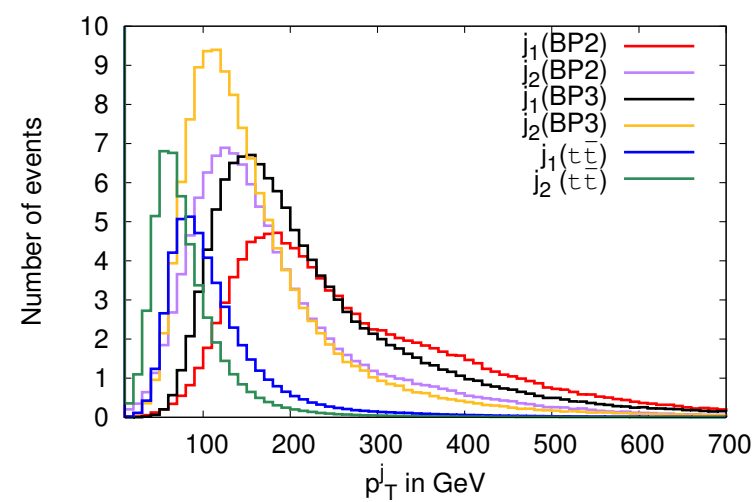

(b)

Figure 3. Lepton and Jet $p_{T}$ distributions coming from BP2 of the $h_{1}^{ \pm} h_{1}^{\mp}$ signal and the SM background $t \bar{t}$ are shown in (a) and (b) respectively.

The non-standard decay products of the light charged Higgs boson $h_{1}^{ \pm}$give rise to gauge bosons $Z, W^{ \pm}$which further decay into charged leptons. Here we tag $e, \mu$ as charged leptons only and $\tau$ is tagged as jet via its hadronic decay. Figure 2 (a) describes the lepton multiplicity distribution coming from BP2 of the $h_{1}^{ \pm} h_{1}^{\mp}$ signal and the SM background $t \bar{t}$. Clearly a higher lepton multiplicity is a winner here. Similarly figure 2 (b) shows the jet multiplicity distributions from BP2 of the $h_{1}^{ \pm} h_{1}^{\mp}$ signal and the SM background $t \bar{t}$. Concerning the signal, these jets are coming from the hadronic decay of $Z, W^{ \pm}, \tau$ as well as the $b$-jets coming from $h_{1}^{ \pm}$. Similarly to the lepton multiplicity, here also we see that the higher jet-multiplicity is preferred for the signal, which can be used to suppress the SM backgrounds.

We keep the cuts in $p_{T}$ of the leptons and the jets relatively low $\left(p_{T} \geq 10 \mathrm{GeV}\right)$, because they will be generated from the lightest pseudoscalar decays. Figure 3 (a) shows the lepton $p_{T}$ distributions for the three leptons in their kinematical order coming from the 


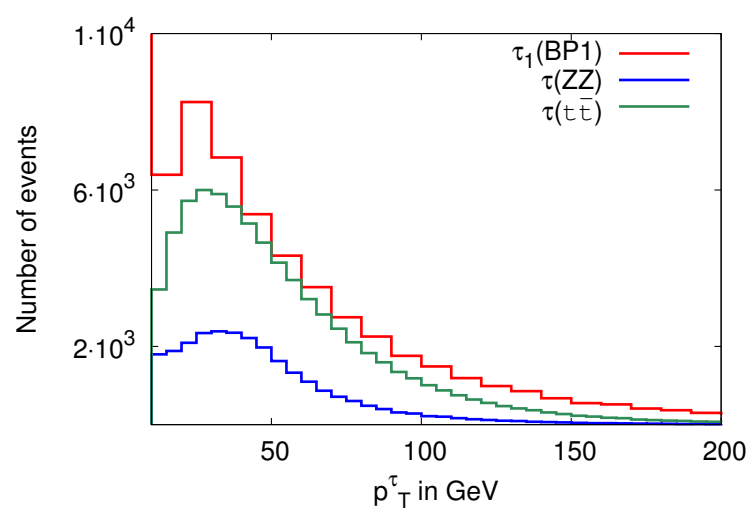

(a)

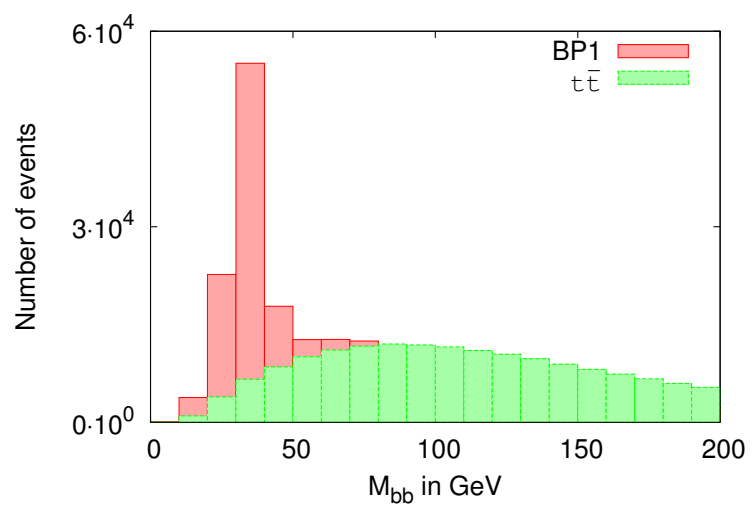

(c)

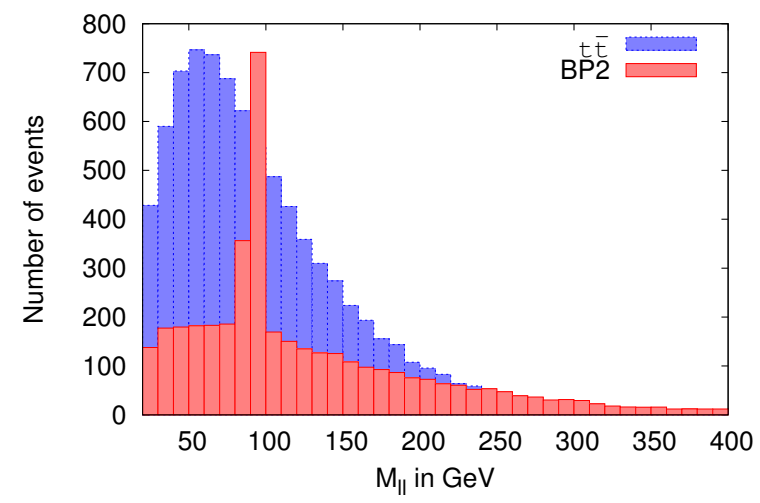

(b)

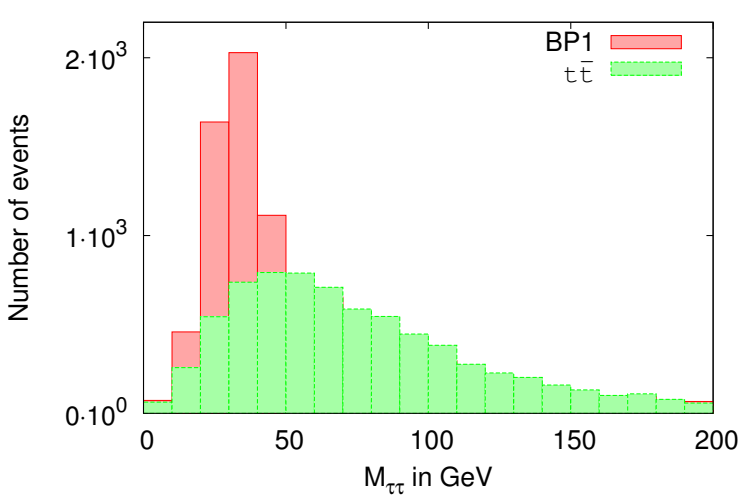

(d)

Figure 4. $\tau-p_{T}$ distributions for $h_{1}^{ \pm} h_{1}^{\mp}$ signal of BP1, $Z Z$ and $t \bar{t}$ are shown in (a). Invariant mass distribution for the (b) di-lepton, (c) di-b-jets, and (d) di-tau coming from BP2 of the $h_{1}^{ \pm} h_{1}^{\mp}$ signal, the SM background $t \bar{t}$ and from BP1 of the $h_{1}^{ \pm} h_{1}^{\mp}$ signal, the SM background $t \bar{t}$ respectively.

signal $h_{1}^{ \pm} h^{\mp}$ for BP2 and from the $t \bar{t}$. The leptons coming from the signals are of high $p_{T}$ because they come from a rather heavy charged Higgs boson for BP2 $\left(m_{h_{1}^{ \pm}} \sim 340 \mathrm{GeV}\right)$, compared to those coming from top quarks. The third lepton coming from the $t \bar{b}$ generally arises from the semi-leptonic $b$ decays and it is rather soft as can be seen from figure 3 (a).

Figure $3(\mathrm{~b})$ describes the jet $p_{T}$ distributions for the first two $p_{T}$ ordered jets coming from BP2 and BP3 of the $h_{1}^{ \pm} h_{1}^{\mp}$ signal and the SM background $t \bar{t}$. We clearly see that a cut $p_{T}>100 \mathrm{GeV}$ on the first jet can reduce such backgrounds considerably. The tagging efficiency of the jet of the b-quark $\left(b_{\text {jet }}\right)$ is obtained through the determination of the secondary vertex and it is therefore momentum dependent. For this purpose we have taken - for the $b_{\text {jet }}$ 's from $t \bar{t}$ — the single-jet tagging efficiency equal to 0.5 , while for the remaining components of the final state we have followed closely the treatment of [52]. Here, in the case of the $\tau_{\text {jet }}$ we have considered the hadronic decay of the $\tau$ to be characterized by at least one charged track with $\Delta R \leq 0.1$ of the candidate $\tau_{\text {jet }}[53,54]$. Figure 4 (a) shows the $\tau_{\text {jet }} p_{T}$ distributions for the BP1 and the dominant SM backgrounds coming from $Z Z, t \bar{t}$. 
Figure 4 (b) describes the invariant mass distribution for the di-lepton coming from BP2 of the $h_{1}^{ \pm} h_{1}^{\mp}$ signal and the SM background $t \bar{t}$. It can be seen that in the case of $t \bar{t}$ both the charged leptons are coming from the corresponding $W^{ \pm}$decays and hence the invariant mass distribution of lepton pair does not have any peak. However, because the charged Higgs boson decays to $Z, W^{ \pm}$, we see the $Z$ mass peak in the invariant mass distribution. The situation is more interesting in the case where $a_{1}$ comes from the charged Higgs decay $h_{1}^{ \pm} \rightarrow a_{1} W^{ \pm}$and then further decays into $b$ or $\tau$ pairs. In this scenario the peak around $m_{a_{1}}$ is easily visible in the variant mass distribution of the di- $b$-jets and $\tau$-jet pair for BP1, as shown in figure 4 (c) and figure 4 (d) respectively. This happens due to the fact that for $\mathrm{BP} 1 \mathcal{B r}\left(h_{1}^{ \pm} \rightarrow a_{1} W^{ \pm} \sim 97 \%\right.$ ) (see table 5 ). We will extract these peaks later to probe such light pseudoscalar.

In oder to distinguish the doublet- and triplet-type of charged Higgs boson we have to find final states with very different predictions for the number of events for the four benchmark points. We shall see in the coming subsections that describes different final states, that it is possible to discriminate between the non-standard decay modes of the charged Higgs boson, i.e. $h_{1}^{ \pm} \rightarrow a_{1} W^{ \pm}$and $h_{1}^{ \pm} \rightarrow Z W^{ \pm}$.

\section{$6.12 b+2 \tau$}

In BP1, BP2 and BP4, the non-standard decay modes i.e. $a_{1} W^{ \pm}$and $Z W^{ \pm}$of the light charged Higgs bosons are open. This results into the possibility to have $\tau$ and $b$ pairs either from $a_{1}$ or $Z$ boson. In this subsection we focus our attention on the final state involving $2 b+2 \tau$. For BP1 the branching ratio into $a_{1} W^{ \pm}$is very high, $\mathcal{B r}\left(h_{1}^{ \pm} \rightarrow a_{1} W^{ \pm} \sim 97 \%\right)$ and thus the possibility of having two $a_{1}$ in the final state is also very high. Such $a_{1}$, produced from the charged Higgs boson decay, mostly decays to $b$ pair $(\sim 95 \%)$ but also goes to $\tau$ pairs $(\sim 5 \%)$, cf. table 4 . Thus $2 b+2 \tau$ is the suitable final state to look for. A scenario like BP1 can be easily probed via this final state where the existence of light pseudoscalar can be also explored.

Table 7 gives the final state numbers with the cumulative cuts for the benchmark points as well as for the dominant SM backgrounds. For the signal, we have included the dominant contributions coming from $h_{1}^{ \pm} h_{1}^{\mp}, h_{2} h_{1}^{ \pm}$and $a_{2} h_{1}^{ \pm}$, whose cross-sections can be found in table 6 . There are many SM processes with final states involving $b$ coming from top quark or $Z$ boson decays and $\tau$ coming from gauge bosons. The dominant SM backgrounds considered are $t \bar{t}, t \bar{t} V, t Z W, V V$ and $V V V$, where $V$ corresponds to $Z$ and/or $W^{ \pm}$bosons. A closer look to the final states will tell us that there are $W^{ \pm}$and $Z$ bosons coming from the charged Higgs and neutral Higgs bosons which can provide additional jets and leptons in the final states. If we tag one of those $W^{ \pm}$boson via $m_{\mathrm{jj}} \sim m_{W}$ with an additional charged lepton ( $e$ or $\mu$ ), then mostly the $h_{1}^{ \pm} h_{1}^{\mp}$ signal will be filtered over the background. In that case the signal significance for BP1 jumped to $7.6 \sigma$ and such signal can be discovered with an integrated luminosity of $43 \mathrm{fb}^{-1}$ at $5 \sigma$ significance. However BP2, BP3 and BP4 will require a much higher integrated luminosity to prove this final state, i.e. 3718,253 and much more than $3000 \mathrm{fb}^{-1}$ respectively. Thus the considered final state can be very effective in the search of the $h_{1}^{ \pm} \rightarrow a_{1} W^{ \pm}$decay mode, which can potentially discover a light pseudoscalar boson. 


\begin{tabular}{|c|c|c|c|c|c|c|c|c|c|}
\hline \multirow{2}{*}{\multicolumn{2}{|c|}{ Final states }} & \multicolumn{4}{|c|}{ Benchmark Points } & \multicolumn{4}{|c|}{ Backgrounds } \\
\hline & & \multirow{2}{*}{$\begin{array}{c}\text { BP1 } \\
52.00\end{array}$} & \multirow{2}{*}{$\begin{array}{c}\mathrm{BP} 2 \\
4.51\end{array}$} & \multirow{2}{*}{$\begin{array}{c}\text { BP3 } \\
2.71\end{array}$} & \multirow{2}{*}{$\begin{array}{l}\mathrm{BP} 4 \\
2.44\end{array}$} & $t \bar{t}$ & $t \bar{t} V$ & $t Z W^{ \pm}$ & $V V / V V V$ \\
\hline \multirow{5}{*}{$2 b+2 \tau$} & $h_{1}^{ \pm} h_{1}^{\mp}$ & & & & & \multirow{5}{*}{24834.00} & \multirow{5}{*}{228.14} & \multirow{5}{*}{11.92} & \multirow{5}{*}{590.91} \\
\hline & $h_{2} h_{1}^{ \pm}$ & 0.48 & 21.75 & 12.84 & 10.60 & & & & \\
\hline & $a_{2} h_{1}^{ \pm}$ & 406.99 & 0.00 & 43.57 & 0.00 & & & & \\
\hline & $t h_{1}^{ \pm}$ & 0.00 & 0.00 & 620.45 & 0.85 & & & & \\
\hline & $t b h_{1}^{ \pm}$ & 0.00 & 0.00 & 25.84 & 0.00 & & & & \\
\hline \multirow{5}{*}{$+1 \ell+m_{\mathrm{jj}} \sim m_{W}$} & $h_{1}^{ \pm} h_{1}^{\mp}$ & 10.75 & 1.02 & 0.25 & 0.50 & \multirow{5}{*}{0.00} & \multirow{5}{*}{25.36} & \multirow{5}{*}{2.91} & \multirow{5}{*}{0.20} \\
\hline & $h_{2} h_{1}^{ \pm}$ & 0.11 & 3.72 & 2.21 & 1.34 & & & & \\
\hline & $a_{2} h_{1}^{ \pm}$ & 67.34 & 0.00 & 8.98 & 0.00 & & & & \\
\hline & $t h_{1}^{ \pm}$ & 0.00 & 0.00 & 7.49 & 0.18 & & & & \\
\hline & $t b h_{1}^{ \pm}$ & 0.00 & 0.00 & 3.47 & 0.00 & & & & \\
\hline \multicolumn{2}{|c|}{ Significance } & $7.60 \sigma$ & $0.82 \sigma$ & $3.14 \sigma$ & $0.37 \sigma$ & & & & \\
\hline \multicolumn{2}{|l|}{$\mathcal{L}_{5 \sigma}\left(\mathrm{fb}^{-1}\right)$} & 43 & 3718 & 253 & $\gg 3000$ & & & & \\
\hline
\end{tabular}

Table 7. The number of events for a $2 b+2 \tau$ final state at $100 \mathrm{fb}^{-1}$ of integrated luminosity at the LHC with $14 \mathrm{TeV}$ center of mass energy.

\section{$6.23 \ell$}

The dominant modes for a triplet-type charged Higgs boson with an existent light pseudoscalar is either $a_{1} W^{ \pm}$or $Z W^{ \pm}$. The final states are, thus, rich in leptons, prompting the multi-leptonic channels. Along with the leptonic final states, there will be $b$-jets coming from the neutral Higgs bosons $\left(a_{1}, a_{2}, h_{2}\right)$ and $Z$ boson decays. Tagging with two such $b$-jets will help to control the SM backgrounds. However, due to the small production cross-sections of such triplet-like charged Higgs bosons, it is possible to probe such final states only at the LHC only with higher integrated luminosity.

In table 8 we present the number of events for the benchmark points and the dominant SM backgrounds with two different kind of selections for the $3 \ell$ final states at $100 \mathrm{fb}^{-1}$ of integrated luminosities at the LHC with $14 \mathrm{TeV}$ center of mass energy. In particular ()$^{\dagger}$ means $p_{T}^{\ell_{2}} \geq 30 \mathrm{GeV}+p_{T}^{\ell_{3}} \geq 40 \mathrm{GeV}$ whereas ()$^{*}$ states that no $p_{T}$ cuts are added. To reduce the background we consider the final state $\left(>3 \ell+>2 j+m_{\ell \ell} \sim m_{Z}\right)^{\dagger}$ and only BP4 can be probed earliest at the LHC, with an integrated luminosity of $437 \mathrm{fb}^{-1}$. The other benchmark points will require a very high luminosity to probe the final state. However, tagging two $b$-jets by choosing $\left(>3 \ell+>2 b_{\mathrm{j}}\right)^{*}$ final state BP1 and BP3 can be probed with much earlier data of 567 and $626 \mathrm{fb}^{-1}$ of integrated luminosity. On the other hand, BP2 and BP4 demand 4216 and $3740 \mathrm{fb}^{-1}$ of integrated luminosities respectively, which are on the edge of the HL-LHC project [55]. Finally, we tag the jet pair coming from the $W^{ \pm}$boson decays by choosing $\left(>3 \ell+m_{\mathrm{jj}} \sim m_{W}\right)^{*}$ final state. In this case the signal number improves a lot for BP4 and it can probed with very early data of $153 \mathrm{fb}^{-1}$ integrated luminosity at the LHC with ECM of $14 \mathrm{TeV}$.

In table 9 we present the case where we tag a tau-jet along with the $\geq 3 \ell$ final states. This makes BP1 and BP4 probable with much earlier data, precisely with 48 and $54 \mathrm{fb}^{-1}$ 


\begin{tabular}{|c|c|c|c|c|c|c|c|c|c|}
\hline \multirow{2}{*}{\multicolumn{2}{|c|}{ Final states }} & \multicolumn{4}{|c|}{ Benchmark Points } & \multicolumn{4}{|c|}{ Backgrounds } \\
\hline & & \multirow{2}{*}{\begin{tabular}{|l|}
$\mathrm{BP} 1$ \\
6.56
\end{tabular}} & \multirow{2}{*}{\begin{tabular}{|l|} 
BP2 \\
8.48 \\
\end{tabular}} & \multirow{2}{*}{\begin{tabular}{|l|} 
BP3 \\
0.06 \\
\end{tabular}} & \multirow{2}{*}{$\begin{array}{c}\text { BP4 } \\
31.41\end{array}$} & $t \bar{t}$ & \multirow[t]{2}{*}{$t \bar{t} V$} & \multirow[t]{2}{*}{$t Z W^{ \pm}$} & $V V / V V V$ \\
\hline \multirow{5}{*}{$(>3 \ell)^{\dagger}$} & $h_{1}^{ \pm} h_{1}^{\mp}$ & & & & & \multirow{5}{*}{0.00} & & & \multirow{5}{*}{4355.69} \\
\hline & $h_{2} h_{1}^{ \pm}$ & 0.02 & 21.35 & 5.80 & 171.21 & & \multirow{4}{*}{348.69} & \multirow{4}{*}{68.14} & \\
\hline & $a_{2} h_{1}^{ \pm}$ & 14.64 & 0.00 & 2.96 & 0.00 & & & & \\
\hline & $t h_{1}^{ \pm}$ & 0.00 & 0.00 & 3.00 & 2.78 & & & & \\
\hline & $t b h_{1}^{ \pm}$ & 0.00 & 0.00 & 1.16 & 0.00 & & & & \\
\hline \multirow{5}{*}{$(+>2 \mathrm{j})^{\dagger}$} & $h_{1}^{ \pm} h_{1}^{\mp}$ & 6.07 & 8.32 & 0.06 & 30.08 & \multirow{5}{*}{0.00} & \multirow{5}{*}{329.52} & \multirow{5}{*}{62.32} & \multirow{5}{*}{2835.27} \\
\hline & $h_{2} h_{1}^{ \pm}$ & 0.03 & 20.64 & 5.76 & 162.07 & & & & \\
\hline & $a_{2} h_{1}^{ \pm}$ & 14.64 & 0.00 & 2.96 & 0.00 & & & & \\
\hline & $t h_{1}^{ \pm}$ & 0.00 & 0.00 & 3.00 & 2.66 & & & & \\
\hline & $t b h_{1}^{ \pm}$ & 0.00 & 0.00 & 1.16 & 0.00 & & & & \\
\hline \multirow{5}{*}{$\left(+m_{\ell \ell} \sim m_{Z}\right)^{\dagger}$} & $h_{1}^{ \pm} h_{1}^{\mp}$ & 3.11 & 5.73 & 0.01 & 24.14 & \multirow{5}{*}{0.00} & \multirow{5}{*}{236.40} & \multirow{5}{*}{59.12} & \multirow{5}{*}{2477.44} \\
\hline & $h_{2} h_{1}^{ \pm}$ & 0.00 & 12.97 & 4.63 & 102.39 & & & & \\
\hline & $a_{2} h_{1}^{ \pm}$ & 2.93 & 0.00 & 1.71 & 0.00 & & & & \\
\hline & $t h_{1}^{ \pm}$ & 0.00 & 0.00 & 0.00 & 2.29 & & & & \\
\hline & $t b h_{1}^{ \pm}$ & 0.00 & 0.00 & 0.39 & 0.00 & & & & \\
\hline \multirow{2}{*}{\multicolumn{2}{|c|}{$\begin{array}{l}\text { Significance } \\
\mathcal{L}_{5 \sigma}\left(\mathrm{fb}^{-1}\right)\end{array}$}} & $0.11 \sigma$ & $0.35 \sigma$ & $0.13 \sigma$ & $2.39 \sigma$ & & & & \\
\hline & & $\gg 3000$ & $\gg 3000$ & $\gg 3000$ & 437 & & & & \\
\hline \multirow{5}{*}{$\left(>3 \ell+>2 b_{\mathrm{j}}\right)^{*}$} & $h_{1}^{ \pm} h_{1}^{\mp}$ & 6.07 & 3.33 & 0.12 & 2.83 & & & & \\
\hline & $h_{2} h_{1}^{ \pm}$ & 0.04 & 11.13 & 6.94 & 11.38 & & & & \\
\hline & $a_{2} h_{1}^{ \pm}$ & 35.14 & 0.00 & 4.81 & 0.00 & 0.00 & 294.55 & 17.74 & 33.01 \\
\hline & $t h_{1}^{ \pm}$ & 0.00 & 0.00 & 25.00 & 1.32 & & & & \\
\hline & $t b h_{1}^{ \pm}$ & 0.00 & 0.00 & 2.31 & 0.00 & & & & \\
\hline Significance & & $2.10 \sigma$ & $0.77 \sigma$ & $2.00 \sigma$ & $0.82 \sigma$ & & & & \\
\hline $\mathcal{L}_{5 \sigma}\left(\mathrm{fb}^{-1}\right)$ & & 567 & 4216 & 626 & 3740 & & & & \\
\hline & $h_{1}^{ \pm} h_{1}^{\mp}$ & 6.91 & 7.73 & 0.02 & 45.90 & & & & \\
\hline & $h_{2} h_{1}^{ \pm}$ & 0.02 & 16.37 & 5.87 & 187.50 & & & & \\
\hline$\left(>3 \ell+m_{\mathrm{jj}} \sim m_{W}\right)^{*}$ & $a_{2} h_{1}^{ \pm}$ & 17.57 & 0.00 & 3.33 & 0.00 & 127.55 & 270.12 & 63.49 & 2804.87 \\
\hline & $t h_{1}^{ \pm}$ & 0.00 & 0.00 & 5.99 & 6.26 & & & & \\
\hline & $t b h_{1}^{ \pm}$ & 0.00 & 0.00 & 0.00 & 0.00 & & & & \\
\hline Significance & & $0.43 \sigma$ & $0.42 \sigma$ & $0.27 \sigma$ & $4.05 \sigma$ & & & & \\
\hline $\mathcal{L}_{5 \sigma}\left(\mathrm{fb}^{-1}\right)$ & & $\gg 3000$ & $\gg 3000$ & $\gg 3000$ & 153 & & & & \\
\hline
\end{tabular}

Table 8. The number of events for a $>3 \ell$ final state at $100 \mathrm{fb}^{-1}$ of integrated luminosity at the LHC with $14 \mathrm{TeV}$ center of mass energy. Here ()$^{\dagger}$ means $p_{T}^{\ell_{2}} \geq 30 \mathrm{GeV}+p_{T}^{\ell_{3}} \geq 40 \mathrm{GeV}$ whereas ( )* states that no $p_{T}$ cuts are added. 


\begin{tabular}{|c|c|c|c|c|c|c|c|c|c|}
\hline \multirow{2}{*}{ Final states } & \multicolumn{3}{|c|}{ Benchmark Points } & \multicolumn{5}{c|}{ Backgrounds } \\
\cline { 2 - 9 } & BP1 & BP2 & BP3 & BP4 & $t \bar{t}$ & $t \bar{t} V$ & $t Z W^{ \pm}$ & $V V / V V V$ \\
\hline \multirow{4}{*}{$3 \ell+1 \tau$} & $h_{1}^{ \pm} h_{1}^{\mp}$ & 95.71 & 19.59 & 0.25 & 83.25 & & & & \\
& $h_{2} h_{1}^{ \pm}$ & 0.24 & 38.05 & 7.08 & 439.47 & & & & \\
& $a_{2} h_{1}^{ \pm}$ & 468.48 & 0.00 & 14.35 & 0.00 & 196.24 & 263.58 & 75.60 & 5009.14 \\
& $t h_{1}^{ \pm}$ & 0.00 & 0.00 & 66.67 & 7.83 & & & & \\
& $t b h_{1}^{ \pm}$ & 0.00 & 0.00 & 23.14 & 0.00 & & & & \\
\hline \multicolumn{2}{|c|}{ Significance } & $7.22 \sigma$ & $0.77 \sigma$ & $1.48 \sigma$ & $6.81 \sigma$ & & & & \\
\multicolumn{2}{|c|}{$\mathcal{L}_{5 \sigma}\left(\mathrm{fb}^{-1}\right)$} & 48 & 4216 & 1138 & 54 & & & & \\
\hline
\end{tabular}

Table 9. The number of events for a $3 \ell+1 \tau$ final state at $100 \mathrm{fb}^{-1}$ of integrated luminosity at the LHC with $14 \mathrm{TeV}$ center of mass energy.

of integrated luminosity respectively. However, such tagging does not help for the other $\mathrm{BPs}$, where the presence of both $a_{1}$ and $W^{ \pm} \mathrm{S}$ are less in the final sate.

\section{$6.3 \quad 3 \tau$}

In this subsection we consider the case where the $\tau \mathrm{s}$ are coming from the pseudoscalars decay and the decays of gauge bosons, viz., $Z$ boson decays into $\tau$ pairs, $W^{ \pm}$boson decays to $\tau \nu$. In this case the multi-tau final state, i.e., $\geq 3 \tau$, is almost free from SM backgrounds. Moreover, if we tag such final state with a charged lepton which arises from the $W^{ \pm}$decay, we reduce more efficiently the residual SM backgrounds. The dominant sources for such final state for $\mathrm{BP} 1$ and $\mathrm{BP} 4$ are $h_{1}^{ \pm} \rightarrow a_{1} W^{ \pm}$and $h_{1}^{ \pm} \rightarrow Z W^{ \pm}$respectively; whereas in the case of BP2 both modes compete, making such final state viable. However, in the case of BP3, such $\tau$ state can only appear when the charged Higgs boson is produced in association of $a_{2}$ or $h_{2}$.

We list the number of the events for $\geq 3 \tau$ and $\geq 3 \tau+\geq 1 \ell$ final states for the benchmark points and the dominant SM backgrounds in table 10. Tagging one charged lepton in the final state makes the gauge boson backgrounds under control. We see that for BP1 the discovery reach can be achieved at $71 \mathrm{fb}^{-1}$ of integrated luminosity. For the other benchmark points it requires higher luminosities, in particular much more than $3000 \mathrm{fb}^{-1}$ for BP2. The final state $\geq 3 \tau+\geq 1 \ell$ will become a possible discovery mode for BP3 and $\mathrm{BP} 4$ only in the case of HL-LHC.

\section{$6.44 \ell$}

Finally we have considered the multi-leptonic final state with $\geq 4 \ell$. The dominant backgrounds are $V V$ and $V V V$, where $V$ corresponds to $Z, W^{ \pm}$bosons. We report in table 11 the number of events for the signal and the dominant backgrounds. We can see that only $\mathrm{BP} 4$ reach a $5 \sigma$ of discovery within the luminosity of LHC, precisely at $81 \mathrm{fb}^{-1}$. Such a discovery can be achieved at the HL-LHC in the case of other BPs. 


\begin{tabular}{|c|c|c|c|c|c|c|c|c|c|}
\hline \multirow{2}{*}{\multicolumn{2}{|c|}{ Final states }} & \multicolumn{4}{|c|}{ Benchmark Points } & \multicolumn{4}{|c|}{ Backgrounds } \\
\hline & & \multirow{2}{*}{$\frac{\mathrm{BP} 1}{26.69}$} & \multirow{2}{*}{$\begin{array}{l}\mathrm{BP} 2 \\
1.62\end{array}$} & \multirow{2}{*}{$\begin{array}{l}\text { BP3 } \\
0.20\end{array}$} & \multirow{2}{*}{$\begin{array}{l}\mathrm{BP} 4 \\
4.22\end{array}$} & $t \bar{t}$ & $t \bar{t} V$ & \multirow[t]{2}{*}{$t Z W^{ \pm}$} & \multirow[t]{2}{*}{$V V / V V V$} \\
\hline \multirow{5}{*}{$\geq 3 \tau$} & $h_{1}^{ \pm} h_{1}^{\mp}$ & & & & & \multirow{5}{*}{0.00} & \multirow{5}{*}{23.42} & & \\
\hline & $h_{2} h_{1}^{ \pm}$ & 0.11 & 5.38 & 1.78 & 19.85 & & & \multirow{4}{*}{4.94} & \multirow{4}{*}{964,12} \\
\hline & $a_{2} h_{1}^{ \pm}$ & 204.96 & 0.00 & 8.52 & 0.00 & & & & \\
\hline & $t h_{1}^{ \pm}$ & 0.00 & 0.00 & 10.00 & 0.47 & & & & \\
\hline & $t b h_{1}^{ \pm}$ & 0.00 & 0.00 & 1.93 & 0.00 & & & & \\
\hline \multirow{5}{*}{$+\geq 1 \ell$} & $h_{1}^{ \pm} h_{1}^{\mp}$ & 7.20 & 0.48 & 0.04 & 1.00 & \multirow{5}{*}{0.00} & \multirow{5}{*}{5.19} & \multirow{5}{*}{0.77} & \multirow{5}{*}{68.67} \\
\hline & $h_{2} h_{1}^{ \pm}$ & 0.03 & 1.55 & 0.30 & 6.36 & & & & \\
\hline & $a_{2} h_{1}^{ \pm}$ & 64.42 & 0.00 & 1.94 & 0.00 & & & & \\
\hline & $t h_{1}^{ \pm}$ & 0.00 & 0.00 & 5.00 & 0.00 & & & & \\
\hline & $t b h_{1}^{ \pm}$ & 0.00 & 0.00 & 0.77 & 0.00 & & & & \\
\hline \multirow{2}{*}{\multicolumn{2}{|c|}{$\begin{array}{l}\text { Significance } \\
\mathcal{L}_{5 \sigma}\left(\mathrm{fb}^{-1}\right)\end{array}$}} & $5.92 \sigma$ & $0.19 \sigma$ & $0.88 \sigma$ & $0.81 \sigma$ & & & & \\
\hline & & 71 & $\gg 3000$ & 3190 & 3784 & & & & \\
\hline
\end{tabular}

Table 10. The number of events for a $>3 \tau$ final state at $100 \mathrm{fb}^{-1}$ of luminosity at the LHC with $14 \mathrm{TeV}$ center of mass energy.

\begin{tabular}{|c|c|c|c|c|c|c|c|c|c|}
\hline \multicolumn{2}{|c|}{ Final states } & \multicolumn{9}{|c|}{ Benchmark Points } & \multicolumn{5}{c|}{ Backgrounds } \\
\cline { 2 - 10 } \multicolumn{2}{|c|}{} & BP1 & BP2 & BP3 & BP4 & $t \bar{t}$ & $t \bar{t} V$ & $t Z W^{ \pm}$ & $V V / V V V$ \\
\hline \multirow{3}{*}{$\geq 4 \ell$} & $h_{1}^{ \pm} h_{1}^{\mp}$ & 14.80 & 11.27 & 0.12 & 44.96 & & & & \\
$+p_{T}^{\ell_{1}} \geq 50 \mathrm{GeV}$ & $h_{2} h_{1}^{ \pm}$ & 0.02 & 27.12 & 2.59 & 255.43 & & & & \\
$+p_{T}^{\ell_{2}} \geq 40 \mathrm{GeV}$ & $a_{1}^{ \pm}$ & 29.28 & 0.00 & 3.24 & 0.00 & 0.00 & 215.85 & 59.13 & 2423.16 \\
& $t h_{1}^{ \pm}$ & 0.00 & 0.00 & 16.67 & 3.50 & & & & \\
& $t b h_{1}^{ \pm}$ & 0.00 & 0.00 & 3.86 & 0.00 & & & & \\
\hline \multicolumn{2}{|c|}{ Significance } & $0.84 \sigma$ & $0.73 \sigma$ & $0.51 \sigma$ & $5.55 \sigma$ & & & & \\
\multicolumn{2}{|c|}{$\mathcal{L}_{5 \sigma}\left(\mathrm{fb}^{-1}\right)$} & 3543 & 4691 & $\gg 3000$ & 81 & & & & \\
\hline
\end{tabular}

Table 11. The number of events for a $4 \ell$ final state at $100 \mathrm{fb}^{-1}$ of luminosity at the LHC with $14 \mathrm{TeV}$ center of mass energy. The $p_{T}$ of the first lepton is greater than $50 \mathrm{GeV}$ whereas the $p_{T}$ of the second lepton is greater than $40 \mathrm{GeV}$. No selections are applied on the other leptons.

\section{Reconstruction of the charged Higgs boson}

In this section we concentrate on the final states appropriate to the respective decay channels which can be used to reconstruct the charged Higgs boson mass, i.e., $m_{h_{1}^{ \pm}}$. We can see from table 5 that for $\mathrm{BP} 1, h_{1}^{ \pm} \rightarrow a_{1} W^{ \pm}$and for $\mathrm{BP} 4, h^{ \pm} \rightarrow Z W^{ \pm}$are the most dominant decay modes, that manifest the existence of light pseudoscalar and the triplet nature of the charged Higgs boson respectively. The light pseudoscalar mass can be reconstructed via the invariant mass of $b$-jet and $\tau$-jet pairs as can be seen from figure $4(\mathrm{c})$ and figure $4(\mathrm{~d})$ 


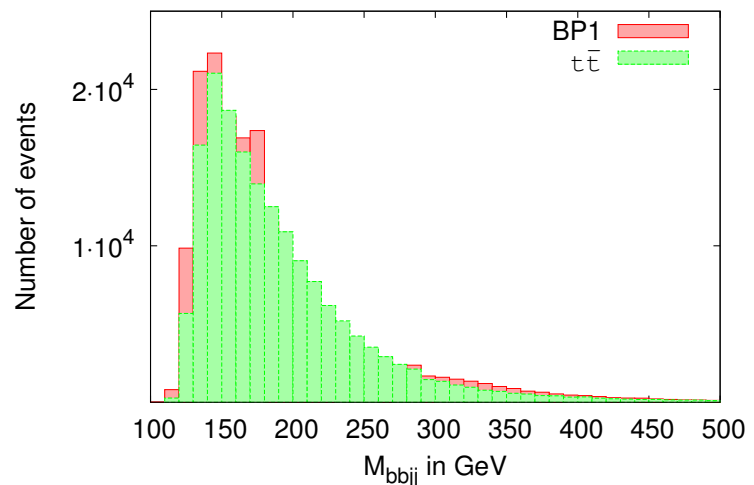

(a)

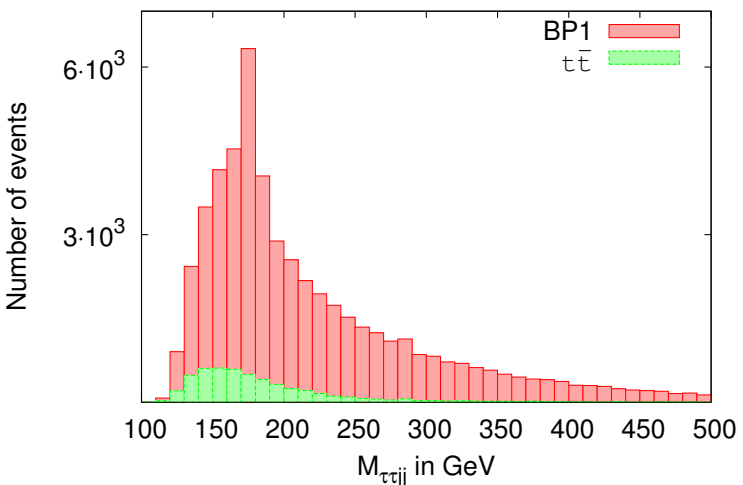

(b)

Figure 5. Invariant mass distributions for the reconstruction of the charged Higgs boson mass for the benchmark points. (a) describes $b b j j$ invariant mass distributions, where $b$-jet pairs are coming from $a_{1}$ peak and normal jet pairs are coming from $W^{ \pm}$peak. (b) shows $\tau \tau j j$ invariant mass distribution where $\tau$-jet pairs are coming from $a_{1}$ mass peak and the normal jet-pairs are coming from $W^{ \pm}$mass peak respectively.

respectively. For this purpose we first reconstruct $a_{1}$ mass peak via $b \bar{b}$ decay mode and we consider $b$-jets within $\pm 7.5 \mathrm{GeV}$ of the $b$-jet invariant mass distribution around the $a_{1}$ mass peak for the reconstruction of the charged Higgs boson. Similarly we reconstruct $W^{ \pm}$mass peak from di-jet invariant mass distribution. Next the $b$-jets from the selected window around the $a_{1}$ peak are taken along with the jets within the $W^{ \pm}$mass window for the $2 b 2 j$ invariant mass reconstruction. Figure 5 (a) shows such distributions for the BP1 together with the dominant SM background $t \bar{t}$. It is clearly visible that for BP1, invariant mass distribution has a local peak around $180 \mathrm{GeV}$, which manifests the charged Higgs boson mass peak.

Next we move to $2 \tau 2 j$ invariant mass distribution, showed in figure $5(\mathrm{~b})$, where the $\tau$-jets are coming from the $\pm 10 \mathrm{GeV}$ window of the $a_{1}$ mass peak and the normal jet pair is taken from $\pm 10 \mathrm{GeV}$ of the $W^{ \pm}$mass window. Clearly we see from figure 5 that $2 \tau 2 j$ peak is much more clear than $2 b 2 j$ but reconstruction of both require very high luminosity run at the LHC. Because $\mathcal{B}\left(a_{1} \rightarrow \tau \bar{\tau}\right) \sim 10 \%$, a better negotiation would be to tag one pair of $b$ coming from the decay of one pseudosclar and one pair of $\tau$ from the other one, where both the pseudoscalars come from the decays of the charged Higgs pair. Here we reconstruct such light pseudoscalar mass peak from the invariant mass distributions of $b$-jet and $\tau$-jet pair.

In table 12 we present the final state numbers for the benchmark points and the dominant SM backgrounds to probe $h^{ \pm} \rightarrow a_{1} W^{ \pm}$decay modes. The significance (required luminosity for $5 \sigma$ discovery) for the benchmark points are given in the last column. The final state is comprised of $\geq 2 \tau-$ jets $+\geq 2 \mathrm{~b}-$ jets $+\geq 1 \ell$, where the $\tau \mathrm{s}$ and $b \mathrm{~s}$ are coming from the light pseudoscalar and one charged lepton can from one of the two $W^{ \pm}$ bosons coming from charged Higgs pair. After that selection events within $\pm 10 \mathrm{GeV}$ of $a_{1}$ mass peak via $b$-jet pair invariant mass have been chosen and presented in table 12 


\begin{tabular}{|c|c|c|c|c|c|c|c|c|c|c|}
\hline & \multicolumn{4}{|c|}{ Signals } & \multicolumn{4}{c|}{ Backgrounds } & Significance \\
\cline { 2 - 10 } & $h_{1}^{ \pm} h_{1}^{\mp}$ & $h_{2} h_{1}^{ \pm}$ & $a_{2} h_{1}^{ \pm}$ & $t h_{1}^{ \pm}$ & $t b h_{1}^{ \pm}$ & $t \bar{t}$ & $t \bar{t} V$ & $t Z W^{ \pm}$ & $V V / V V V$ & $\mathcal{L}_{5 \sigma}\left(\mathrm{fb}^{-1}\right)$ \\
\hline BP1 & 6.81 & 0.04 & 23.42 & 0.00 & 0.00 & 0.00 & 1.41 & 0.01 & 0.10 & $5.37 \sigma(87)$ \\
BP2 & 0.07 & 0.34 & 0.00 & 0.00 & 0.00 & 0.00 & 1.57 & 0.01 & 0.10 & $0.28 \sigma(\gg 3000)$ \\
BP3 & 0.01 & 0.19 & 0.56 & 0.00 & 0.39 & 0.00 & 1.20 & 0.01 & 0.00 & $0.75 \sigma(4471)$ \\
BP4 & 0.17 & 0.45 & 0.00 & 0.02 & 0.00 & 0.00 & 2.15 & 0.01 & 0.00 & $0.40 \sigma(\gg 3000)$ \\
\hline
\end{tabular}

Table 12. The number of event combination of $b$-jet pair at $100 \mathrm{fb}^{-1}$ integrated luminosity, where $b$ jets are within $\pm 10 \mathrm{GeV}$ of $a_{1}$ mass peak in a final state comprised of $\geq 2 \tau-$ jets $+\geq 2 \mathrm{~b}-$ jets $+\geq 1 \ell$. The significance (required luminosity for $5 \sigma$ discovery) for the benchmark points are given in the last column.

\begin{tabular}{|c|c|c|c|c|c|c|c|c|c|c|}
\hline & \multicolumn{5}{|c|}{ Signals } & \multicolumn{4}{|c|}{ Backgrounds } & \multirow{2}{*}{$\begin{array}{l}\text { Significance } \\
\mathcal{L}_{5 \sigma}\left(\mathrm{fb}^{-1}\right)\end{array}$} \\
\hline & $h_{1}^{ \pm} h_{1}^{\mp}$ & $h_{2} h_{1}^{ \pm}$ & $a_{2} h_{1}^{ \pm}$ & $t h_{1}^{ \pm}$ & $t b h_{1}^{ \pm}$ & $t \bar{t}$ & $t \bar{t} V$ & $t Z W^{ \pm}$ & $V V / V V V$ & \\
\hline BP1 & 7.79 & 0.06 & 38.06 & 0.00 & 0.00 & 0.00 & 0.58 & 0.00 & 0.00 & $6.73 \sigma(55)$ \\
\hline $\mathrm{BP} 2$ & 0.10 & 1.03 & 0.00 & 0.00 & 0.00 & 0.00 & 0.37 & 0.00 & 0.00 & $0.92 \sigma(2937)$ \\
\hline BP3 & 0.18 & 0.79 & 3.43 & 4.50 & 0.00 & 0.00 & 0.58 & 0.00 & 0.00 & $2.89 \sigma(299)$ \\
\hline $\mathrm{BP} 4$ & 0.00 & 0.45 & 0.00 & 0.01 & 0.00 & 0.00 & 0.74 & 0.01 & 0.00 & $0.42 \sigma(\gg 3000)$ \\
\hline
\end{tabular}

Table 13. The number of event combination of $\tau$-jet pair at $100 \mathrm{fb}^{-1}$ integrated luminosity, where $\tau$ jets are within $\pm 10 \mathrm{GeV}$ of $a_{1}$ mass peak in a final state comprised of $\geq 2 \tau-$ jets $+\geq 2 \mathrm{~b}-$ jets $+\geq 1 \ell$. The significance (required luminosity for $5 \sigma$ discovery) for the benchmark points are given in the last column.

at an integrated luminosity $100 \mathrm{fb}^{-1}$. It can be seen that only for BP1, whose dominant decay mode is $h^{ \pm} \rightarrow a_{1} W^{ \pm}$, a discovery of $5.37 \sigma$ can be achieved at $100 \mathrm{fb}^{-1}$ of integrated luminosity. For other benchmark points one needs very high luminosities, as can be read from table 12.

Similarly table 13 presents the final states comprised of $\geq 2 \tau-$ jets $+\geq 2 \mathrm{~b}-$ jets $+\geq 1 \ell$, where the $\tau \mathrm{s}$ and $b \mathrm{~s}$ are coming from the light pseudoscalar and one charged lepton come from one of the two $W^{ \pm}$bosons coming from charged Higgs pair. However, in this case we select the events for which the $\tau$-jet pairs falls within $\pm 10 \mathrm{GeV}$ of $a_{1}$ mass peak. The significance at an integrated luminosity of $100 \mathrm{fb}^{-1}$ (required luminosity for $5 \sigma$ discovery) for the benchmark points are given in the last column of table 13. In this case also BP1 reaches a signal significance of $6.73 \sigma$ whereas for the other benchmark points a discovery of $5 \sigma$ requires higher luminosities.

We now move to reconstruct the charged Higgs mass via its triplet nature, i.e. via $h_{1}^{ \pm} \rightarrow Z W^{ \pm} \rightarrow 2 l 2 j$. The presence of multiple charged leptons makes the final state much cleaner and can be probed with relatively earlier data. Figure 6 shows the invariant mass distribution of di-lepton and di-jet, i.e. $m_{\ell \ell j j}$, where the di-leptons are coming from the $Z$ boson and are selected within $\pm 5 \mathrm{GeV}$ of $Z$ mass peak of the di-lepton invariant mass distribution $\left(m_{\ell \ell}\right)$ and di-jets are coming from the $W$ boson, which are selected when they fall within $\pm 10 \mathrm{GeV}$ of the di-jet invariant mass distribution $m_{j j}$. We can see that for BP4 


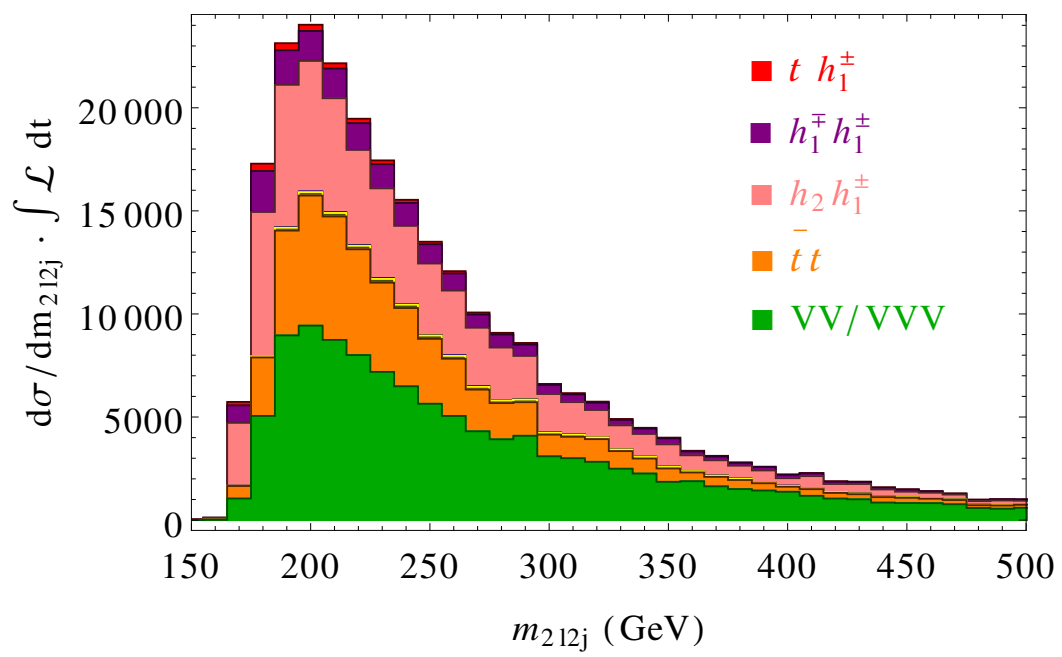

Figure 6. Invariant mass distribution $m_{2 \ell 2 j}$ for BP 4 and the dominant SM backgrounds at $100 \mathrm{fb}^{-1}$ of integrated luminosity. The number of events for the signals $h_{1}^{ \pm} h_{1}^{\mp}, h_{2} h_{1}^{ \pm}$and $t h_{1}^{ \pm}$are multiplied by a factor of 50 for the sake of the presentation.

\begin{tabular}{|c|c|c|c|c|c|c|c|c|c|c|}
\hline & \multicolumn{5}{|c|}{ Signals } & \multicolumn{4}{c|}{ Backgrounds } & \multirow{2}{*}{$\begin{array}{c}\text { Significance } \\
\end{array}$} \\
\cline { 2 - 9 } & $h_{1}^{ \pm} h_{1}^{\mp}$ & $h_{2} h_{1}^{ \pm}$ & $a_{2} h_{1}^{ \pm}$ & $t h_{1}^{ \pm}$ & $t b h_{1}^{ \pm}$ & $t \bar{t}$ & $t \bar{t} V$ & $t Z W^{ \pm}$ & $V V / V V V$ & $\mathcal{L}_{5 \sigma}\left(\mathrm{fb}^{-1}\right)$ \\
\hline BP1 & 1.09 & 0.00 & 8.78 & 0.00 & 0.00 & 29.44 & 20.69 & 4.36 & 450.65 & $0.43 \sigma(\gg 3000)$ \\
BP2 & 0.77 & 2.03 & 0.00 & 0.00 & 0.00 & 9.81 & 15.52 & 3.49 & 87.24 & $0.26 \sigma(\gg 3000)$ \\
BP3 & 0.00 & 0.57 & 0.14 & 1.50 & 0.00 & 9.81 & 18.00 & 4.56 & 148.70 & $0.16 \sigma(\gg 3000)$ \\
BP4 & 5.83 & 31.12 & 0.00 & 1.27 & 0.00 & 19.62 & 15.26 & 3.39 & 339.84 & $1.87 \sigma(712)$ \\
\hline
\end{tabular}

Table 14. The number of event combination for $m_{\ell \ell j j}$ at $100 \mathrm{fb}^{-1}$ integrated luminosity, where $\ell$ are within $\pm 5 \mathrm{GeV}$ of $Z$ mass peak and normal jets are within $\pm 10 \mathrm{GeV}$ of $W^{ \pm}$mass peak.

it is possible to achieve the reconstructed charged Higgs mass peak via $h_{1}^{ \pm} \rightarrow Z W^{ \pm}$mode. Table 14 shows the reconstructed event combinations within $\pm 10 \mathrm{GeV}$ of the charged Higgs mass peak for the benchmark points and the corresponding total SM background numbers. It is clearly seen that only for BP4 a discovery of $5 \sigma$ can be achieved below $1000 \mathrm{fb}^{-1}(712)$ of integrated luminosity. For the rest of the points one needs very high luminosity run of LHC $\left(\gg 3000 \mathrm{fb}^{-1}\right)$. Thus a perfectly triplet-like singly charged Higgs boson can be easily probed via $Z W^{ \pm}$decay modes, which is not possible for a doublet-like or a mixed charged Higgs boson at the LHC run-I.

\section{Distinguishing from other extended scenario}

In the previous sections we have discussed the theoretical and phenomenological aspects of a charged Higgs boson in the context of the TNMSSM. This model is characterized by the presence of a light pseudoscalar in the spectrum, which allows the decay channel $h_{1}^{ \pm} \rightarrow a_{1} W^{ \pm}$, as well as by the triplet-like decay channel $h_{1}^{ \pm} \rightarrow Z W^{ \pm}$. The light pseudoscalar, together with the $h^{ \pm} \rightarrow a W^{ \pm}$decay mode, is also present in NMSSM [11] and its 


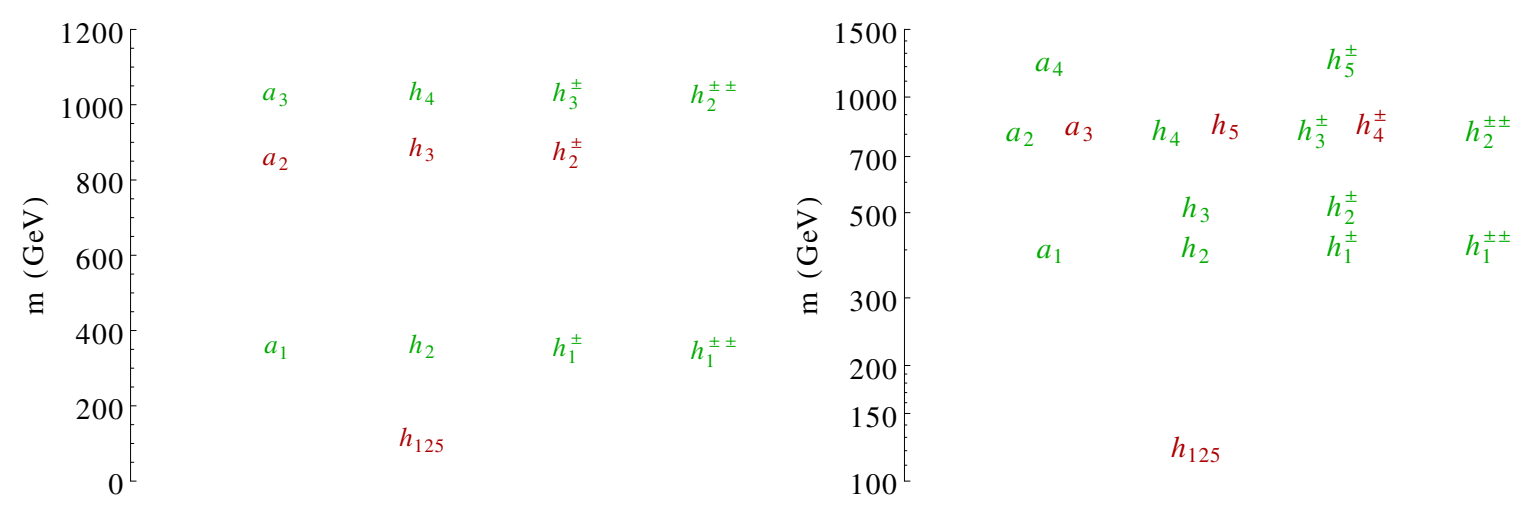

(a)

(b)

Figure 7. A typical mass hierarchy of the scalar sector, with the doublets in red and the triplet Higgs bosons in green color. We have considered an extension of the MSSM with a $Y= \pm 1$ triplet (a) and the custodial limit of an extension of the MSSM with both $Y=0$ and $Y= \pm 1$ triplets (b).

phenomenology has been well studied for a doublet-like charged Higgs boson [56-58]. Similarly the presence of $Z W^{ \pm}$decay demonstrates a triplet-like charged Higgs boson which breaks custodial symmetry $[5,10]$. However, TNMSSM gives an opportunity to have both the decay modes. This enables us to straightway separate the models from completely doublet-type charged Higgs boson(s) and completely triplet-type charged Higgs bosons.

We have considered various benchmark points in order to probe these two different decay modes of the lightest charged Higgs boson and it has been found that both $h^{ \pm} \rightarrow a W^{ \pm}$ and $h_{1}^{ \pm} \rightarrow Z W^{ \pm}$are unlikely to happen for a parameter point. Confronting table 2 and table 6 we see that, apart from the pair production cross section, the relevant production channel for a triplet charged Higgs boson is either in association with $a_{2}$ or $h_{2}$. The associated production cross-sections for both $\mathrm{BP} 1$ and $\mathrm{BP} 4$ are the same, $\sim 300 \mathrm{fb}$, because of the apparent mass degeneracy among $h_{1}^{ \pm}$and $a_{2} / h_{2}$ respectively.

The tendency of the gauge representation to group in the same mass value for scalar, pseudoscalar and charged Higgs bosons was pointed out recently [23]. Here we want to emphasize that this is true even in the case of other possible triplet extensions of the MSSM. In figure 7 we present the scalar mass spectrum for two different triplet extensions of the MSSM. In figure 7 (a) we have considered the extension of the MSSM with a $Y= \pm 1$ triplet superfield [8], whereas in figure 7 (b) the case where both the $Y=0$ and $Y= \pm 1$ triplet superfields (custodial triplets) are present [10]. The phenomenology of the $Y= \pm 1$ triplet was studied in [59] whereas the model with triplets in the custodial symmetric limit was analyzed in $[60,61]$. We have selected the two sample points scanning over the parameter space and requesting the presence the doublet-type lightest scalar Higgs boson $h_{1} \equiv h_{125}$ with a tree-level mass $\sim 125 \mathrm{GeV}$. In both the case there are two doubly-charged charged Higgs bosons in the spectrum, because of the presence of the $Y= \pm 1$ triplets. We can see that these doubly-charged states are degenerate in mass with one of the triplet neutral scalars, pseudoscalars and one of the triplet-like singly-charged Higgs bosons. Such scenarios thus claim to have one doubly charged Higgs boson in the similar mass range 
of the triplet-like singly charged Higgs boson and finding both will surely shed light on the existence of the multiple $\mathrm{SU}(2)_{L}$ triplets in the spectrum including the one with nonzero hypercharge. The doubly charged Higgs boson phenomenologies are independently studied in $[8,10,59-61]$. Thus finding a triplet-like singly charged Higgs boson and one neutral scalar $\left(a_{2} / h_{2}\right)$ with the same mass but no doubly charged Higgs bosons is a proof of existence of a $Y=0$ triplet in the spectrum. However, the existence of mass degenerate triplet-like doubly charged Higgs boson with or without additional neutral scalars along with the triplet-like singly charged Higgs boson in the mass spectrum surely tells about the existence of multiple $\mathrm{SU}(2)_{L}$ triplets, i.e. $Y= \pm 1$ and $Y=0, \pm 1$ respectively. The search modes discussed in this article separate doublet- and triplet-like singly charged Higgs bosons with standard or non-standard decay modes. On top of the mass degeneracy information, existence/non-existence of a doubly charged Higgs boson will give us addition handle to pin down about the others gauge representation in the Higgs potential that plays a crucial role in electro-weak symmetry breaking.

The Higgs phenomenology of the extended Higgs sector in non-supersymmetric models is very similar to the supersymmetric case. The existence of the light pseudoscalar, i.e. the pseudo-Nambu-Goldstone boson, of course will be governed by the breaking of the global U(1) symmetry of the scalar potential. Similar to the supersymmetric case this corresponds to small Higgs trilinear couplings $A_{i} H_{i}^{\dagger} T H_{i}$ [65], which breaks this global symmetry. There are very limited analysis for triplet-like light pseudoscalar as pNGB mode for non-supersymmetric theories, however there are plenty of studies on the Higgs phenomenology with different hypercharged triplet [66-70].

\section{Conclusions}

In this article we prescribe some search modes for light singly charged Higgs boson via which we can shed light on the gauge representation of the charged Higgs bosons. A triplet-like charged Higgs boson does not couple to fermions, thus neither it is possible to produce it via fermionic modes nor it decays in the fermionic modes, i.e. $\tau \nu$ and/or $t b$. However a triplet-charged Higgs boson couples to $Z W^{ \pm}$, which gives rise to additional production and decay modes. We explore these features in order to separate a triplet-like singly charged Higgs boson from a doublet one, even in the presence of a light-pseudoscalar, which gives rise to an additional decay mode, i.e. $a_{1} W^{ \pm}$.

We have analyzed signatures of a supersymmetric extension of the SM, characterized by an extra $Y=0$ Higgs triplet and a SM gauge singlet, in view of the recent and previous Higgs data. We have chosen different benchmark points which represent different decay modes preferred either by a triplet-like or by a doublet-like charged Higgs boson. In particular, BP3 represents a completely doublet-like charged Higgs boson and BP4 represents a completely triple-like charged Higgs bosons. In the case of $\mathrm{BP} 1, a_{1} W^{ \pm}$mode is the most dominant, whereas in BP2 we have mixed scenario. We have seen that a BP1 like scenario with dominant decay mode in $a_{1} W^{ \pm}$can be probed at the LHC with $14 \mathrm{TeV}$ of ECM with very early data of $\sim 43 \mathrm{fb}^{-1}$ integrated luminosity via $2 b+2 \tau+1 \ell+m_{j j} \sim m_{W}$ final state. 
The discovery of such light pseudoscalar can be achieved with very early data of $\sim$ $55 \mathrm{fb}^{-1}$ and this would be certainly a signal in favor of an extended Higgs sectors. The NMSSM does have such light pseudoscalar but does not have any extra charged Higgs bosons compared to the MSSM, while the TNMSSM has an extra triplet-like charged Higgs boson. This possibility, changes the direct bounds derived from searches for a charged Higgs at the LHC, as well as the indirect bounds on flavour. These changes are due to the doublet-triplet mixing in the charged Higgs and chargino sectors of the triplet extended model [62-64].

The triplet-like charged Higgs boson and its decay mode to $Z W^{ \pm}$can be probed via $3 \ell+1 \tau$ with an early data of $\sim 54 \mathrm{fb}^{-1}$ of integrated luminosity. The charged Higgs mass can be reconstructed with relatively larger data of $\sim 712 \mathrm{fb}^{-1}$. Finding such triplet-charged state would clearly be proof of the existence of higher representation of $\mathrm{SU}(2)_{L}$ in the Higgs potential. We have also presented the sensitivity in other possible search modes.

The existence of non-zero hypercharge triplets would require to have more than one doubly charged Higgs boson degenerate along with the singlet triplet-like charged Higgs bosons. Finding such doubly charged states are necessary in order to prove the existence of the triplet representation with non-zero hypercharge.

Open Access. This article is distributed under the terms of the Creative Commons Attribution License (CC-BY 4.0), which permits any use, distribution and reproduction in any medium, provided the original author(s) and source are credited.

\section{References}

[1] ATLAS collaboration, Observation of a new particle in the search for the Standard Model Higgs boson with the ATLAS detector at the LHC, Phys. Lett. B 716 (2012) 1 [arXiv: 1207.7214] [INSPIRE].

[2] CMS collaboration, Observation of a new boson at a mass of $125 \mathrm{GeV}$ with the CMS experiment at the LHC, Phys. Lett. B 716 (2012) 30 [arXiv:1207.7235] [INSPIRE].

[3] A. Djouadi, The anatomy of electro-weak symmetry breaking. II. The Higgs bosons in the minimal supersymmetric model, Phys. Rept. 459 (2008) 1 [hep-ph/0503173] [INSPIRE].

[4] J.F. Gunion and C. Hays, Higgs triplets, CERN-2006-009.497 [INSPIRE].

[5] S. Di Chiara and K. Hsieh, Triplet Extended Supersymmetric Standard Model, Phys. Rev. D 78 (2008) 055016 [arXiv:0805.2623] [INSPIRE].

[6] T. Basak and S. Mohanty, Triplet-Singlet Extension of the MSSM with a $125 \mathrm{GeV}$ Higgs and Dark Matter, Phys. Rev. D 86 (2012) 075031 [arXiv:1204.6592] [INSPIRE].

[7] Particle Data Group collaboration, J. Beringer et al., Review of Particle Physics (RPP), Phys. Rev. D 86 (2012) 010001 [inSPIRE].

[8] K. Agashe, A. Azatov, A. Katz and D. Kim, Improving the tunings of the MSSM by adding triplets and singlet, Phys. Rev. D 84 (2011) 115024 [arXiv:1109.2842] [INSPIRE].

[9] H. Georgi and M. Machacek, Doubly Charged Higgs Bosons, Nucl. Phys. B 262 (1985) 463 [INSPIRE].

[10] L. Cort, M. Garcia and M. Quirós, Supersymmetric Custodial Triplets, Phys. Rev. D 88 (2013) 075010 [arXiv:1308.4025] [INSPIRE]. 
[11] U. Ellwanger, C. Hugonie and A.M. Teixeira, The Next-to-Minimal Supersymmetric Standard Model, Phys. Rept. 496 (2010) 1 [arXiv:0910.1785] [InSPIRE].

[12] P. Bandyopadhyay, C. Corianò and A. Costantini, Perspectives on a supersymmetric extension of the standard model with a $Y=0$ Higgs triplet and a singlet at the LHC, JHEP 09 (2015) 045 [arXiv: 1506.03634] [INSPIRE].

[13] ATLAS collaboration, Search for charged Higgs bosons produced in association with a top quark and decaying via $H^{ \pm} \rightarrow \tau \nu$ using pp collision data recorded at $\sqrt{s}=13 \mathrm{TeV}$ by the ATLAS detector, Phys. Lett. B 759 (2016) 555 [arXiv:1603.09203] [INSPIRE].

[14] ATLAS collaboration, Search for charged Higgs bosons in the $H^{ \pm} \rightarrow t b$ decay channel in $p p$ collisions at $\sqrt{s}=13 \mathrm{TeV}$ using the ATLAS detector, ATLAS-CONF-2016-089 (2016).

[15] CMS collaboration, Search for a light charged Higgs boson decaying to $\mathrm{c} \overline{\mathrm{s}}$ in $p p$ collisions at $\sqrt{s}=8 \mathrm{TeV}$, JHEP 12 (2015) 178 [arXiv:1510.04252] [INSPIRE].

[16] CMS collaboration, Search for charged Higgs bosons with the $\mathrm{H}^{ \pm} \rightarrow \tau^{ \pm} \nu_{\tau}$ decay channel in the fully hadronic final state at $\sqrt{s}=13 \mathrm{TeV}$, CMS-PAS-HIG-16-031.

[17] ALEPH, DELPHI, L3, OPAL collaborations, LEP Working Group for Higgs Boson Searches, R. Barate et al., Search for the standard model Higgs boson at LEP, Phys. Lett. B $\mathbf{5 6 5}$ (2003) 61 [hep-ex/0306033] [INSPIRE].

[18] ALEPH, DELPHI, L3, OPAL collaborations, LEP Working Group for Higgs Boson Searches, S. Schael et al., Search for neutral MSSM Higgs bosons at LEP, Eur. Phys. J. C 47 (2006) 547 [hep-ex/0602042] [INSPIRE].

[19] P. Franzini et al., Limits on Higgs Bosons, Scalar Quarkonia and $\eta(B)$ 's From Radiative $\Upsilon$ Decays, Phys. Rev. D 35 (1987) 2883 [inSPIRE].

[20] LHCb, CMS collaborations, Observation of the rare $B_{s}^{0} \rightarrow \mu^{+} \mu^{-}$decay from the combined analysis of CMS and LHCb data, Nature $\mathbf{5 2 2}$ (2015) 68 [arXiv:1411.4413] [INSPIRE].

[21] P. Bandyopadhyay, K. Huitu and A. Sabanci Keceli, Multi-Lepton Signatures of the Triplet Like Charged Higgs at the LHC, JHEP 05 (2015) 026 [arXiv: 1412.7359] [INSPIRE].

[22] J.L. Diaz-Cruz, J. Hernandez-Sanchez, S. Moretti and A. Rosado, Charged Higgs boson phenomenology in Supersymmetric models with Higgs triplets, Phys. Rev. D 77 (2008) 035007 [arXiv: 0710 . 4169] [INSPIRE].

[23] P. Bandyopadhyay, C. Corianò and A. Costantini, General analysis of the charged Higgs sector of the $Y=0$ triplet-singlet extension of the MSSM at the LHC, Phys. Rev. D 94 (2016) 055030 [arXiv: 1512.08651] [INSPIRE].

[24] P. Bandyopadhyay, C. Corianò and A. Costantini, Probing the hidden Higgs bosons of the $Y=0$ triplet- and singlet-extended Supersymmetric Standard Model at the LHC, JHEP 12 (2015) 127 [arXiv: 1510.06309] [INSPIRE].

[25] K. Huitu, J. Maalampi, A. Pietila and M. Raidal, Doubly charged Higgs at LHC, Nucl. Phys. B 487 (1997) 27 [hep-ph/9606311] [INSPIRE].

[26] M. Muhlleitner and M. Spira, A Note on doubly charged Higgs pair production at hadron colliders, Phys. Rev. D 68 (2003) 117701 [hep-ph/0305288] [INSPIRE].

[27] A.G. Akeroyd and M. Aoki, Single and pair production of doubly charged Higgs bosons at hadron colliders, Phys. Rev. D 72 (2005) 035011 [hep-ph/0506176] [INSPIRE].

[28] K.S. Babu and S. Jana, Probing Doubly Charged Higgs Bosons at the LHC through Photon Initiated Processes, Phys. Rev. D 95 (2017) 055020 [arXiv: 1612.09224] [INSPIRE]. 
[29] E. Asakawa and S. Kanemura, The $H^{ \pm} W^{\mp} Z^{0}$ vertex and single charged Higgs boson production via $W Z$ fusion at the large hadron collider, Phys. Lett. B 626 (2005) 111 [hep-ph/0506310] [INSPIRE].

[30] ATLAS, CMS collaborations, Combined Measurement of the Higgs Boson Mass in pp Collisions at $\sqrt{s}=7$ and $8 \mathrm{TeV}$ with the ATLAS and CMS Experiments, Phys. Rev. Lett. 114 (2015) 191803 [arXiv: 1503.07589] [INSPIRE].

[31] CMS collaboration, Measurement of Higgs boson production and properties in the $W W$ decay channel with leptonic final states, JHEP 01 (2014) 096 [arXiv:1312.1129] [INSPIRE].

[32] CMS collaboration, Measurement of the properties of a Higgs boson in the four-lepton final state, Phys. Rev. D 89 (2014) 092007 [arXiv:1312.5353] [INSPIRE].

[33] ATLAS collaboration, Measurements of the Higgs boson production and decay rates and coupling strengths using pp collision data at $\sqrt{s}=7$ and $8 \mathrm{TeV}$ in the ATLAS experiment, ATLAS-CONF-2015-007 (2015).

[34] ATLAS collaboration, Measurements of Higgs boson production and couplings in the four-lepton channel in pp collisions at center-of-mass energies of 7 and $8 \mathrm{TeV}$ with the ATLAS detector, Phys. Rev. D 91 (2015) 012006 [arXiv:1408.5191] [ATLAS-CONF-2015-007] [INSPIRE].

[35] ATLAS collaboration, Observation and measurement of Higgs boson decays to $W W^{*}$ with the ATLAS detector, Phys. Rev. D 92 (2015) 012006 [arXiv:1412.2641] [INSPIRE].

[36] ATLAS collaboration, Measurement of the Higgs boson mass from the $H \rightarrow \gamma \gamma$ and $H \rightarrow Z Z^{*} \rightarrow 4 \ell$ channels with the ATLAS detector using $25 \mathrm{fb}^{-1}$ of pp collision data, Phys. Rev. D 90 (2014) 052004 [arXiv: 1406.3827] [INSPIRE].

[37] ATLAS collaboration, ATLAS Run 1 searches for direct pair production of third-generation squarks at the Large Hadron Collider, Eur. Phys. J. C 75 (2015) 510 [arXiv:1506.08616] [INSPIRE].

[38] CMS collaboration, Searches for third-generation squark production in fully hadronic final states in proton-proton collisions at $\sqrt{s}=8 \mathrm{TeV}$, JHEP 06 (2015) 116 [arXiv:1503.08037] [INSPIRE].

[39] http://lepsusy.web.cern.ch/lepsusy/www/inos_moriond01/charginos_pub.html.

[40] CMS collaboration, Search for charged Higgs bosons with the $H^{+} \rightarrow \tau^{+} \nu_{\tau}$ decay channel in the fully hadronic final state at $\sqrt{s}=8 \mathrm{TeV}$, CMS-PAS-HIG-14-020.

[41] CMS collaboration, Search for a heavy charged Higgs boson in proton-proton collisions at $\sqrt{s}=8 \mathrm{TeV}$ with the CMS detector, CMS-PAS-HIG-13-026.

[42] ATLAS collaboration, Search for neutral Higgs bosons of the minimal supersymmetric standard model in pp collisions at $\sqrt{s}=8 \mathrm{TeV}$ with the ATLAS detector, JHEP 11 (2014) 056 [arXiv: 1409.6064] [INSPIRE].

[43] ATLAS collaboration, Search for strongly produced superpartners in final states with two same sign leptons with the ATLAS detector using $21 \mathrm{fb}^{-1}$ of proton-proton collisions at $\sqrt{s}=8 \mathrm{TeV}$, ATLAS-CONF-2013-007.

[44] F. Staub, SARAH 3.2: Dirac Gauginos, UFO output and more, Comput. Phys. Commun. 184 (2013) 1792 [arXiv:1207.0906] [inSPIRE].

[45] A. Pukhov, CalcHEP 2.3: MSSM, structure functions, event generation, batchs and generation of matrix elements for other packages, hep-ph/0412191 [INSPIRE]. 
[46] A. Datta, A. Djouadi, M. Guchait and Y. Mambrini, Charged Higgs production from SUSY particle cascade decays at the CERN LHC, Phys. Rev. D 65 (2002) 015007 [hep-ph/0107271] [INSPIRE].

[47] A. Djouadi, The Anatomy of electro-weak symmetry breaking. II. The Higgs bosons in the minimal supersymmetric model, Phys. Rept. 459 (2008) 1 [hep-ph/0503173] [INSPIRE].

[48] T. Sjöstrand, L. Lönnblad and S. Mrenna, PYTHIA 6.2: Physics and manual, hep-ph/0108264 [INSPIRE].

[49] P.Z. Skands et al., SUSY Les Houches accord: Interfacing SUSY spectrum calculators, decay packages and event generators, JHEP 07 (2004) 036 [hep-ph/0311123] [INSPIRE].

[50] http://skands.physics.monash.edu/slha/.

[51] M. Cacciari, G.P. Salam and G. Soyez, FastJet User Manual, Eur. Phys. J. C 72 (2012) 1896 [arXiv:1111.6097] [INSPIRE].

[52] I.R. Tomalin, b tagging in CMS, J. Phys. Conf. Ser. 110 (2008) 092033 [InSPIRE].

[53] G. Bagliesi, Tau tagging at ATLAS and CMS, arXiv:0707.0928 [INSPIRE].

[54] CMS collaboration, CMS technical design report, volume II: Physics performance, J. Phys. G 34 (2007) 995 [INSPIRE].

[55] http://hilumilhc.web.cern.ch/.

[56] B. Coleppa, F. Kling and S. Su, Charged Higgs search via $A W^{ \pm} / H W^{ \pm}$channel, JHEP 12 (2014) 148 [arXiv: 1408.4119] [INSPIRE].

[57] F. Kling, A. Pyarelal and S. Su, Light Charged Higgs Bosons to AW/HW via Top Decay, JHEP 11 (2015) 051 [arXiv: 1504.06624] [INSPIRE].

[58] P. Bandyopadhyay, K. Huitu and S. Niyogi, Non-standard charged Higgs decay at the LHC in Next-to-Minimal Supersymmetric Standard Model, JHEP 07 (2016) 015 [arXiv:1512.09241] [INSPIRE].

[59] M. Frank, D.K. Ghosh, K. Huitu, S.K. Rai, I. Saha and H. Waltari, Left-right supersymmetry after the Higgs boson discovery, Phys. Rev. D 90 (2014) 115021 [arXiv:1408.2423] [INSPIRE].

[60] C.-W. Chiang, S. Kanemura and K. Yagyu, Phenomenology of the Georgi-Machacek model at future electron-positron colliders, Phys. Rev. D 93 (2016) 055002 [arXiv:1510.06297] [INSPIRE].

[61] C.-W. Chiang and A.-L. Kuo, Can the 750-GeV diphoton resonance be the singlet Higgs boson of custodial Higgs triplet model?, Phys. Lett. B 760 (2016) 634 [arXiv:1601.06394] [INSPIRE].

[62] P. Bandyopadhyay, K. Huitu and A. Sabanci Keceli, Multi-Lepton Signatures of the Triplet Like Charged Higgs at the LHC, JHEP 05 (2015) 026 [arXiv:1412.7359] [INSPIRE].

[63] P. Bandyopadhyay, S. Di Chiara, K. Huitu and A.S. Keçeli, Naturality vs perturbativity, $B_{s}$ physics and LHC data in triplet extension of MSSM, JHEP 11 (2014) 062 [arXiv: 1407.4836] [INSPIRE].

[64] P. Bandyopadhyay, K. Huitu and A. Sabanci, Status of $Y=0$ Triplet Higgs with supersymmetry in the light of $\sim 125$ GeV Higgs discovery, JHEP 10 (2013) 091 [arXiv: 1306.4530] [INSPIRE].

[65] P. Bandyopadhyay and A. Costantini, Phenomenology of triplet extended Standard Model at the $L H C$, in preparation. 
[66] H.E. Logan and M.-A. Roy, Higgs couplings in a model with triplets, Phys. Rev. D 82 (2010) 115011 [arXiv: 1008.4869] [INSPIRE].

[67] S. Blunier, G. Cottin, M.A. Díaz and B. Koch, Phenomenology of a Higgs triplet model at future $e^{+} e^{-}$colliders, Phys. Rev. D 95 (2017) 075038 [arXiv:1611.07896] [INSPIRE].

[68] J.F. Gunion, R. Vega and J. Wudka, Higgs triplets in the standard model, Phys. Rev. D 42 (1990) 1673 [INSPIRE].

[69] T. Araki, C.Q. Geng and K.I. Nagao, Signatures of Dark Matter in Inert Triplet Models, Int. J. Mod. Phys. D 20 (2011) 1433 [arXiv:1108.2753] [InSPIRE].

[70] C. Englert, E. Re and M. Spannowsky, Triplet Higgs boson collider phenomenology after the LHC, Phys. Rev. D 87 (2013) 095014 [arXiv: 1302.6505] [INSPIRE]. 